\title{
Bounds of Functional Outputs for Parabolic Problems Part II: Bounds of the Excat Solution
}

\author{
N. Parés \\ P. Díez \\ A. Huerta
}




\section{Bounds of Functional Outputs for Parabolic Problems Part II: Bounds of the Excat Solution}
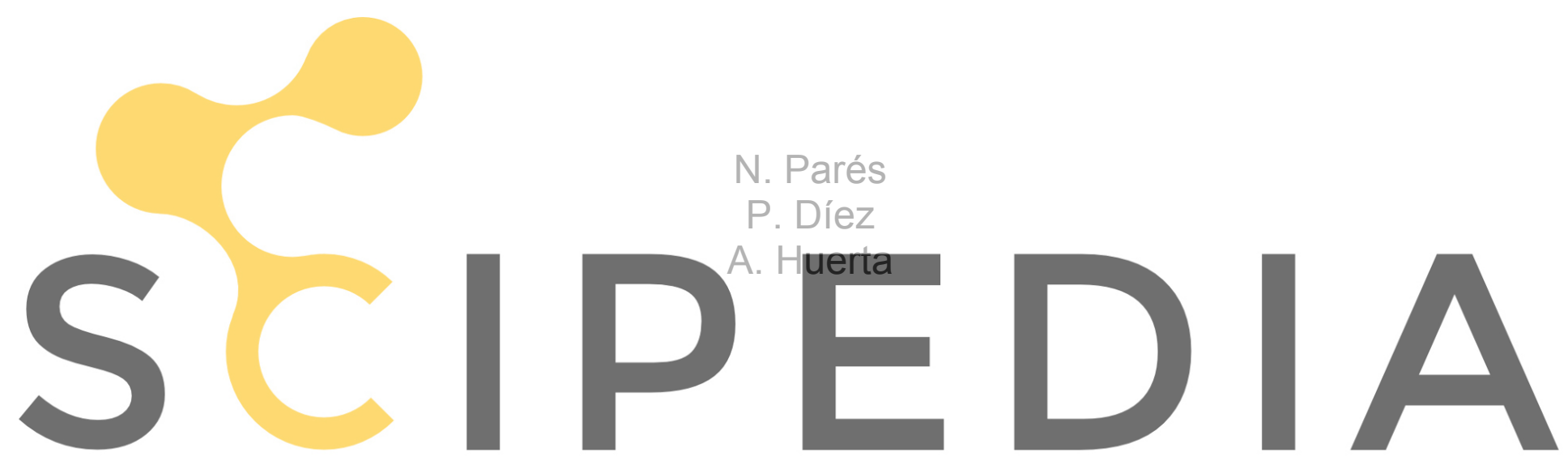

Register for free at https//www.scipedia.com to download the version without the watermark

\section{Publication CIMNE №-299, January 2007}




\title{
Bounds of functional outputs for parabolic problems. Part II: Bounds of the exact solution ${ }^{*}$
}

\author{
Núria Parés, Pedro Díez and Antonio Huerta \\ Laboratori de Càlcul Numèric (LaCàN) \\ Universitat Politècnica de Catalunya \\ e-mail: nuria.pares,pedro.diez,antonio.huerta@upc.edu
}

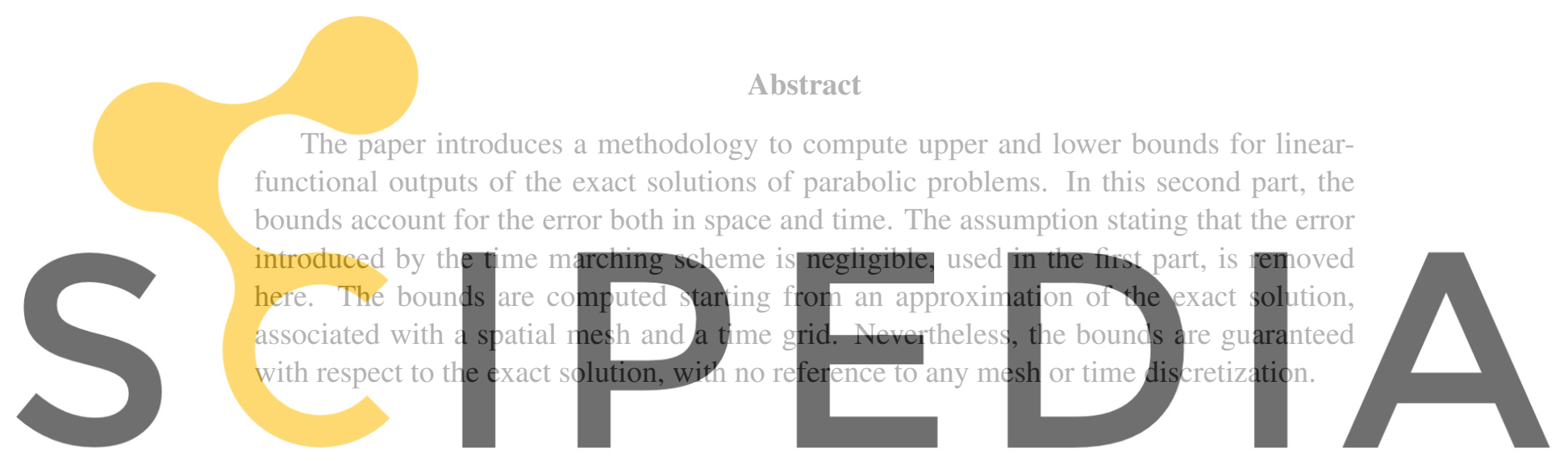

1 Introduction

Register for free at https//www.scipedia.com to download the version without the watermark

Goal-oriented adaptive strategies and the related error assessment techniques have been exten-

sively studied for steady elliptic problems [13, 12, 2, 4, 9]. The error in the quantity of interest is assessed combining upper and lower bounds for the energy norm of the original problem (primal) and of an auxiliary problem (adjoint) associated with the selected output. The bounds of the quantity of interest are readily obtained operating with the energy estimates. Most of these tools provide asymptotic bounds that is with respect to a much finer reference discretization. Other approaches focus on guarantying exact bounds, that is bounds guaranteed with respect to the exact solution, independently of any underlying reference mesh. The motivation to develop these numerical tools is to certify the accuracy of the solutions of boundary value and/or evolution problems, see [17, 18, 14, 22].

This paper and its associated first part [15] provide a methodology to obtain computable strict bounds for quantities of interest in the context of parabolic problems. In this context, the list of previous references is much shorter $[1,3,19,10,5]$. The strategy presented here uses ideas from $[10,18,7]$ and produces exact bounds for linear-functional outputs accounting both for the error arising from the space and time discretization. Note that in the first part [15] the effect of the time discretization is neglected. The error information and the bounds are used in an adaptive procedure where both $h$ and $\Delta t$ (space mesh size and time step) are adapted.

${ }^{\dagger}$ Submitted to Computer Methods in Applied Mechanics and Engineering 
The methodology presented here takes as input two continuous (both in space and time) approximate solutions of both the direct advection-reaction-diffusion problem and the adjoint problem associated with the selected output. In the application examples, these approximations are obtained post-processing the approximations given by the discontinuous Galerkin method in time, that is smoothing out the time discontinuities. Actually, any other method providing a piecewise continuous polynomial function both in space and time may be used. The methodology presented here works out the space-time residual error equations and reduces the problem of finding bounds of the output of interest to properly combine the solutions of a number of steady (time-independent) problems where the standard methods are applicable. Thus, computable bounds are derived using a strategy based on the ideas given in [18], which allows producing bounds for steady problems, to a series of steady reaction-diffusion problems.

\section{Problem statement}

\subsection{Model problem}

The transient convection-reaction-diffusion equation is considered in $\Omega \subset \mathbb{R}^{n_{\text {sd }}}$, where $n_{\text {sd }}$ is the number of spatial dimensions and $\Omega$ is polygonal for $n_{\mathrm{sd}}=2$ and polyhedric for $\mathrm{n}_{\mathrm{sd}}=3$. The time interval of interest is $I=] 0, T]$. For the sake of a simple presentation, it is assumed that all the boundary conditions are of Dirichlet type and homogeneous. Thus, the weak solution $u$ is such that for each $t \in I, u(t) \in \mathcal{H}\left(\frac{1}{0}(\Omega):=\mathcal{V}\right.$, where $\mathcal{H}_{0}^{1}(\Omega)$ denotes the standard Soboley space of functions vanis
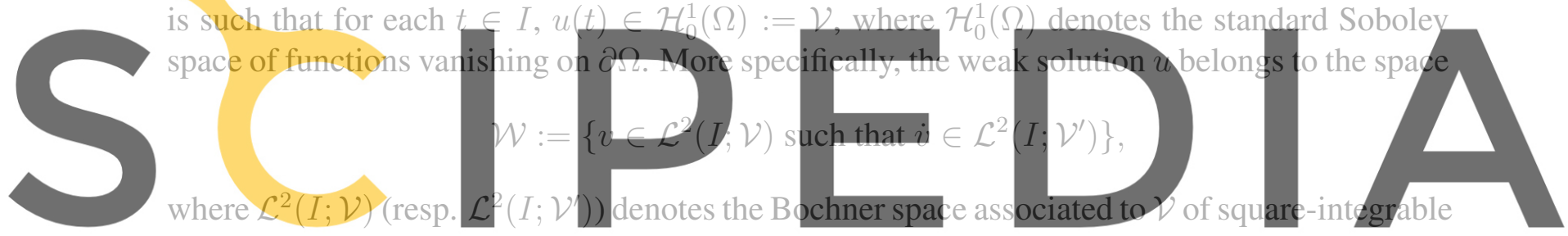

functions from $I$ into $\mathcal{V}$ (resp. $\left.\mathcal{V}^{\prime}\right)$

Register for free at https//www.scipedia.com to download the $e_{\Gamma}$ version without the watermark $\mathcal{L}^{2}(I ; \mathcal{V}):=\left\{v: I \rightarrow \mathcal{V}, v(t)\right.$ is $\mathcal{V}$-measurable and $\left.\int_{0}\|v(t)\|_{\nu}^{2} \mathrm{~d} t<+\infty\right\}$,

$\|\cdot\|_{\mathcal{V}}$ being the norm associated with $\mathcal{V},\|\cdot\|_{\mathcal{V}}=\|\cdot\|_{1}$.

The weak (both in space and time) variational form of the problem is: find $u \in \mathcal{W}$ such that

$$
A(u, v)=L(v) \quad \forall v \in \mathcal{L}^{2}(I ; \mathcal{V}),
$$

for

$$
A(w, v):=\int_{0}^{T}[\langle\dot{w}, v\rangle+a(t ; w, v)] \mathrm{d} t+(w(0), v(0))
$$

and

$$
L(v):=\int_{0}^{T} \ell(t ; v) \mathrm{d} t+\left(u_{0}, v(0)\right),
$$

where $\langle\cdot, \cdot \cdot\rangle$ denotes the duality pairing between $\mathcal{V}^{\prime}$ and $\mathcal{V},(\cdot, \cdot)$ denotes the $L^{2}(\Omega)$ inner product and $u_{0}$ is the initial condition weakly imposed.

Here, $u_{0} \in \mathcal{V}$ and the forms $a(t ; \cdot, \cdot)$ and $\ell(t ; \cdot)$ are

$$
a(t ; w, v):=\int_{\Omega}[\nu(t) \boldsymbol{\nabla} w \cdot \boldsymbol{\nabla} v+\boldsymbol{\alpha}(t) \cdot \boldsymbol{\nabla} w v+\sigma(t) w v] \mathrm{d} \Omega,
$$


and

$$
\ell(t ; v):=\langle f(t), v\rangle=\int_{\Omega} f(t) v \mathrm{~d} \Omega
$$

where $f \in \mathcal{L}^{2}\left(I ; \mathcal{V}^{\prime}\right)$ and for each $t \in I, \nu(t) \in \mathcal{L}^{\infty}(\Omega)$ is a strictly positive real coefficient, $\sigma(t) \in \mathcal{L}^{\infty}(\Omega)$ is a nonnegative real coefficient and $\boldsymbol{\alpha}(t) \in \mathcal{H}($ div; $\Omega)$ is a prescribed vector field which is assumed for simplicity to be divergence-free, $\boldsymbol{\nabla} \cdot \boldsymbol{\alpha}(t)=0$, that is $\boldsymbol{\alpha}$ is a velocity field of an incompressible flow. Moreover $\nu, \sigma$ and $\boldsymbol{\alpha}$ are assumed to be sufficiently smooth in time.

\subsection{Continuous approximation}

The exact solution of the boundary value problem (1) has to be approximated. In the following, the approximation of $u, u_{\tau, h}^{\mathrm{sm}}$, is assumed to be continuous both in space and time. Note that if the method provides a discontinuous approximation, it has to be smoothed out in order to fulfill this assumption.

The approximation $u_{\tau, h}^{\mathrm{sm}}$ is associated with a spatial mesh of the domain $\Omega$ and to a timegrid discretization of $I$. The characteristic element size of the mesh is denoted by $h$ and the characteristic time step is denoted by $\tau$. The space mesh generates a discrete space $\mathcal{V}^{h} \subset \mathcal{V}$. The points of the time grid are denoted by $0=t^{0}<t^{1}<\ldots<t^{n}<\ldots<t^{N}=T$ and the corresponding time slabs are denoted by $I_{n}=\left(t^{n-1}, t^{n}\right]$.

The approximation $u_{\tau, h}^{\mathrm{sm}}$ is piecewise polynomial in time; that is, $u_{\tau, h}^{\mathrm{sm}}$ is polynomial of de-

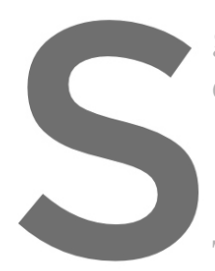
gree $q$ inside each time $\operatorname{slab} I_{n}$ and gipbally continuous.
dependence is such that $u_{\tau, h}^{\mathrm{sm}}(t) \in \mathcal{V}^{h}$. That is,
\[ u_{\tau, h}^{\mathrm{sm}} \in \mathcal{W}^{\tau, h}:=\left\{v \in \mathcal{C}^{0}(\overline{\mathcal{Y}} ; \mathcal{V}),\left.v\right|_{I_{n}} \in \mathbb{P}^{q}\left(I_{n} ; \nu\right.\right. \]
The time-polynomial snace $\mathbb{P}\left(\boldsymbol{I} ; \mathcal{V}^{h}\right)$ is defined in terms of

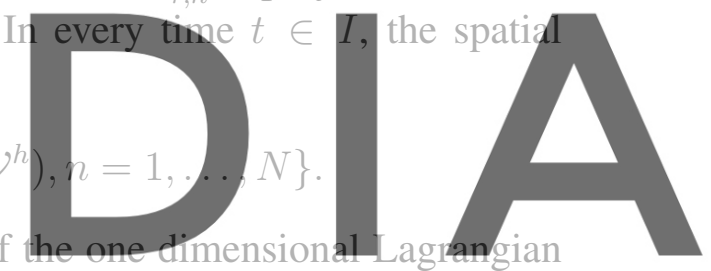

shape functions of degree $q$ in the interval $I_{n}, N_{n j}(\cdot), j=0, \ldots, q$

Register for free at https//www.scipedia.com to download the version without the watermark

$$
\mathbb{P}^{q}\left(I_{n} ; \nu^{h}\right):=\left\{v: I_{n} \rightarrow \nu^{h}, v(t)=\sum_{j=0}^{q} v_{j} N_{n j}(t), v_{j} \in \nu^{h}\right\} .
$$

It is important to note that equation (1) only imposes the initial condition weakly; however, the smoothed approximation $u_{\tau, h}^{\mathrm{sm}}$ must verifiy the initial condition exactly, namely $u_{\tau, h}^{\mathrm{sm}}(0)=$ $u_{0}$. This is only possible if $u_{0} \in \mathcal{V}^{h} \subset \mathcal{V}$ and therefore this has to be also included as an assumption.

In this work, the approximation $u_{\tau, h}^{\mathrm{sm}}$ is obtained postprocessing the approximation of $u$ provided by the discontinuous Galerkin method in time, $\mathrm{cG}(p) \mathrm{dG}(q)$. The $\mathrm{cG}(p) \mathrm{dG}(q)$ approximation of $u$ is a standard continuous Galerkin finite element approximation of degree $p$ in space (where $p$ denotes the degree of the complete polynomials used in the interpolation of $\mathcal{V}^{h}$ ) and it is a piecewise polynomial globally discontinuous Galerkin approximation of degree $q$ in time $[20,6,21]$. Thus, the continuous space-time approximation required here, $u_{\tau, h}^{\mathrm{sm}}$, is recovered by a simple post-processing, smoothing out the time-discontinuities at $t=t^{n}, n=1 \ldots N-1$. It is worth noting, however, that the method presented here is valid for any approximation of $u$ in $\mathcal{W}^{\tau, h}$ such that $u_{\tau, h}^{\text {sm }}(0)=u_{0}$.

In order to simplify the notation, in the remainder of the paper the dependence on the time discretization is omitted and, consequently, the continuous approximation $u_{\tau, h}^{\mathrm{sm}}$ and the associated interpolation space $\mathcal{W}^{\tau, h}$ are denoted by $u_{h}$ and $\mathcal{W}^{h}$ respectively. 


\subsection{Error equation}

The equation for the error associated with $u_{h}, e=u-u_{h} \in \mathcal{W}$, is obtained replacing $u=e+u_{h}$ in equation (1) and using the linearity of the first argument of $A(\cdot, \cdot)$. Thus, $e \in \mathcal{W}$ is such that

$$
A(e, v)=L(v)-A\left(u_{h}, v\right)=: R^{\mathrm{P}}(v) \quad \forall v \in \mathcal{L}^{2}(I ; \mathcal{V}),
$$

where $R^{\mathrm{P}}(\cdot)$ is the residual associated with the approximation $u_{h}$. It is worth noting that, since $u_{h}$ is not a Galerkin approximation of $u$, then, the Galerkin orthogonality condition of the residual does not hold in general, that is

$$
R^{\mathrm{P}}(v) \text { is not necessarily } 0 \quad \forall v \in \mathcal{W}^{h} .
$$

\section{Outputs of interest and adjoint problem}

As previously said, this paper aims at providing upper and lower bounds for quantities of interest depending on the exact solution $u$. Here, the quantities of interest are restricted to be linear functions and therefore they take the form

$$
L^{\mathcal{O}}(u)=\int_{0}^{T} \ell^{\mathcal{O}}(t ; u) \mathrm{d} t+\left(u_{T}^{\mathcal{O}}, u(T)\right),
$$

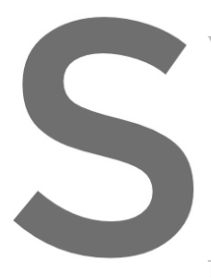

where $u_{T}^{O} \in \nu^{k}$ and
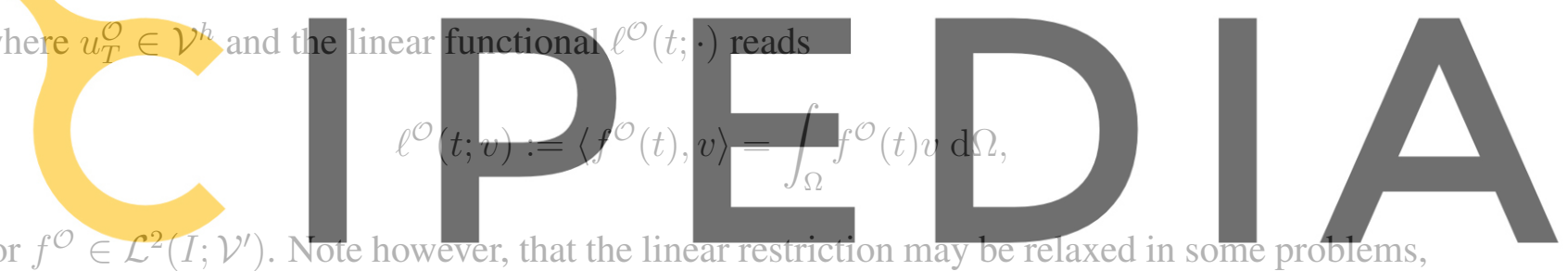

see $[22]$

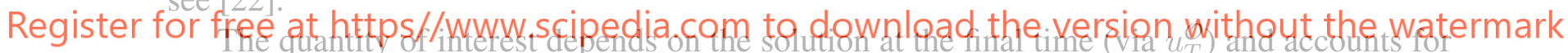

the behavior of the solution along the complete time evolution (via the weight function $f^{\mathcal{O}}$ ).

Due to the linearity of $L^{\mathcal{O}}(\cdot)$, assessing the value or obtaining bounds for $L^{\mathcal{O}}(u)$ is equivalent to evaluate or bound $L^{\mathcal{O}}(e)$. In other words, introducing $s:=L^{\mathcal{O}}(u)-L^{\mathcal{O}}\left(u_{h}\right)=L^{\mathcal{O}}(e)$ and computing bounds for $s$,

$$
s^{l b} \leq s \leq s^{u b},
$$

is perfectly equivalent to compute bounds for $L^{\mathcal{O}}(u)$ :

$$
L^{\mathcal{O}}\left(u_{h}\right)+s^{l b} \leq L^{\mathcal{O}}(u) \leq L^{\mathcal{O}}\left(u_{h}\right)+s^{u b} .
$$

An adjoint (or dual) problem with respect to the selected output is introduced in order to derive upper and lower bounds for $s$. The adjoint problem reads: find $\psi \in \mathcal{V}$ such that

$$
A(v, \psi)=L^{\mathcal{O}}(v) \quad \forall v \in \mathcal{W} .
$$

Note that following the definition in (5) the initial condition for $\psi$ is now $u_{T}^{\mathcal{O}}$ at $t=T$.

Analogous to the direct (or primal) problem, the adjoint problem is solved numerically. Similarly to the primal problem, the smoothed approximation to the dual problem, $\psi_{h}$, is continuous both in space an time, belongs to $\mathcal{W}^{h}$ and verifies exactly the "initial" condition $\psi_{h}(T)=u_{T}^{\mathcal{O}}$. 
The error associated with the adjoint approximation $\psi_{h}$ is $\varepsilon:=\psi-\psi_{h} \in \mathcal{W}$, and it is such that

$$
A(v, \varepsilon)=L^{\mathcal{O}}(v)-A\left(v, \psi_{h}\right)=: R^{\mathrm{D}}(v) \quad \forall v \in \mathcal{W},
$$

where $R^{\mathrm{D}}(\cdot)$ is the weak adjoint residual associated with $\psi_{h}$. Also here, the adjoint residual does not fulfill, in general, the Galerkin orthogonality condition

$$
R^{\mathrm{D}}(v) \text { is not necessarily } 0 \quad \forall v \in \mathcal{W}^{h} .
$$

\section{Bounding the output by a space-time norm}

This section introduces bounds of the output of interest $s$ in terms of a space-time norm denoted by $\||\cdot|\|$. The choice of the norm is the same as in [10]. This choice is not unique. In fact other authors $[1,3]$ use different measures.

For every time $t$, the inner spatial product associated with the symmetric counterpart of the bilinear form $a(t ; \cdot, \cdot)$ is introduced

$$
v):=\frac{1}{2}(a(t ; w, v)+a(t ; v, w))=\int_{\Omega}[\nu(t) \nabla w \cdot \nabla v+\sigma(t) w v] \mathrm{d} \Omega .
$$

Note that the advection term (related to $\alpha(t)$ ) is purely skew-symmetric because $\alpha(t)$ is divergencefree $\forall t \in I$ and the boundary conditions are of Dirichlet type. This inner product induces the norm denoted by $\|\cdot\|,\|v\|^{2}:=a^{\mathrm{s}}(t ; v, v)=a(t ; v, v)$. The space-time norm $\|\cdot\|$ is readily defined as

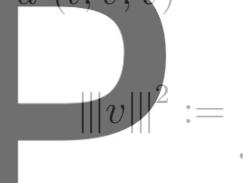

and the

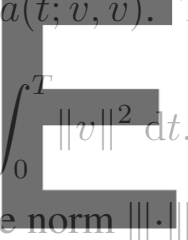

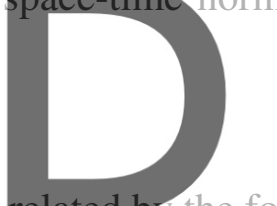

are related by the following

The proof is straightforward from the definition of $A(\cdot, \cdot)$. See also the particularization of

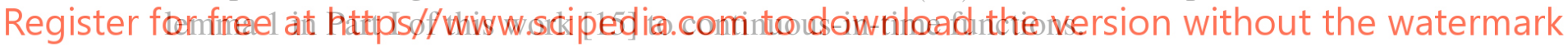

Lemma 1 For any $v \in \mathcal{W}$

$$
A(v, v)=\|\| v\left\|^{2}+\frac{1}{2}(v(0), v(0))+\frac{1}{2}(v(T), v(T)) \geq\right\| v\|\|^{2} .
$$

The following result shows that bounding $s$ is equivalent to obtain upper bounds for the errors measured in the space-time norm $\||\cdot|\|$.

Theorem 1 Let $e^{s}$ and $\varepsilon^{s} \in \widehat{\mathcal{W}}$ be such that for any $v \in \mathcal{W}$

$$
\int_{0}^{T} a^{\mathrm{s}}\left(t ; e^{s}, v\right) \mathrm{d} t=R^{P}(v) \quad \text { and } \quad \int_{0}^{T} a^{\mathrm{s}}\left(t ; \varepsilon^{s}, v\right) \mathrm{d} t=R^{D}(v)
$$

where

$$
\widehat{\mathcal{W}}:=\left\{v \in \mathcal{L}^{2}(I ; \mathcal{V}),\left.v\right|_{I_{n}} \in \mathcal{L}^{2}\left(I_{n} ; \mathcal{V}\right) \text { and }\left.\dot{v}\right|_{I_{n}} \in \mathcal{L}^{2}\left(I_{n} ; \mathcal{V}^{\prime}\right)\right\}
$$

Then,

$$
R^{P}\left(\psi_{h}\right)-\frac{1}{4}\|\| \kappa e^{s}-\frac{1}{\kappa} \varepsilon^{s}\|\|^{2} \leq s \leq R^{P}\left(\psi_{h}\right)+\frac{1}{4}\left\|\kappa e^{s}+\frac{1}{\kappa} \varepsilon^{s}\right\| \|^{2},
$$

for any nonzero parameter $\kappa \in \mathbb{R}$. 
Proof. Combining equation (7) for $v=e$ and equation (3) for $v=\psi_{h}$ yields the following error representation

$$
s=L^{\mathcal{O}}(e)=L^{\mathcal{O}}(e)-A\left(e, \psi_{h}\right)+A\left(e, \psi_{h}\right)=R^{\mathrm{D}}(e)+R^{\mathrm{P}}\left(\psi_{h}\right),
$$

where the term $R^{\mathrm{P}}\left(\psi_{h}\right)$ is not zero, in general, since the Galerkin orthogonality property of the primal residual does not hold, see equation (4).

Also, taking $v=e \in \mathcal{W}$ in equation (3) and using the relation between the bilinear form $A(\cdot, \cdot)$ and $\|\mid \cdot\|$ given in lemma 1 it follows that

$$
R^{\mathrm{P}}(e)=A(e, e) \geq\|\| \|^{2} .
$$

The proof now follows from a simple algebraic manipulation. Indeed, let $\kappa$ be a nonzero real parameter and consider the inequality

$$
\left\|\frac{1}{2}\left(\kappa e^{s} \pm \frac{1}{\kappa} \varepsilon^{s}\right)-\kappa e\right\|^{2} \geq 0 .
$$

\section{Expansion of the 1.h.s. yields}

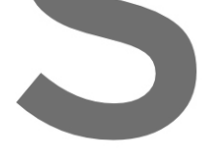

\section{Moreover, using $v=e$

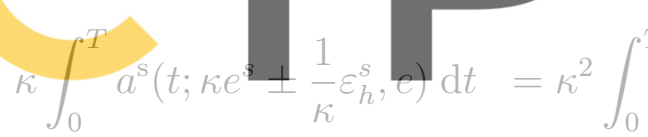

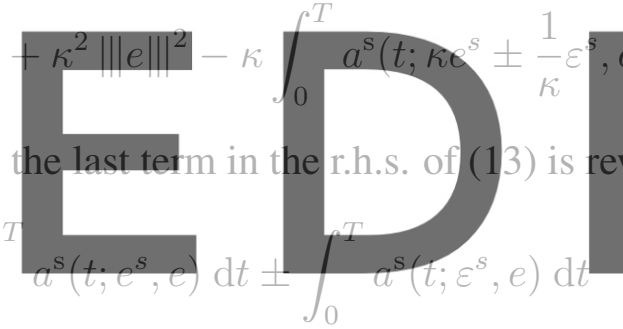

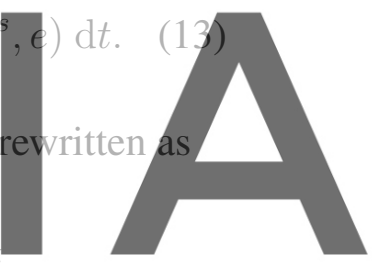

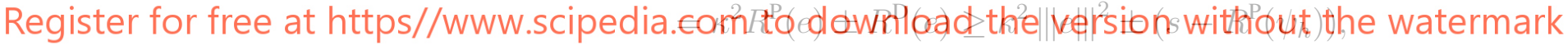

where equations (10) and (11) are used to derive the inequality.

Considering equations (12) and (13) yields

$$
0 \leq\left\|\frac{1}{2}\left(\kappa e^{s} \pm \frac{1}{\kappa} \varepsilon^{s}\right)-\kappa e\right\|\left\|^{2} \leq \mp s \pm R^{\mathrm{P}}\left(\psi_{h}\right)+\frac{1}{4}\right\|\left\|e^{s} \pm \frac{1}{\kappa} \varepsilon^{s}\right\| \|^{2},
$$

that is,

$$
\pm s \leq \pm R^{\mathrm{P}}\left(\psi_{h}\right)+\frac{1}{4}\left\|\kappa e^{s} \pm \frac{1}{\kappa} \varepsilon^{s}\right\| \|^{2} .
$$

The proof is concluded by noting that the + sign in the previous equation yields the expression for the upper bound of $s$, whereas the - sign yields the expression for the lower bound of $s$.

Theorem 1 reveals that bounds for $s$ are obtained if the space-time norms of the linear combinations of $e^{s}$ and $\varepsilon^{s}$ are available. It follows also that it is sufficient to obtain upper bounds of these norms, namely

$$
R^{\mathrm{P}}\left(\psi_{h}\right)-\frac{1}{4}\left|\left\|\kappa e^{s}-\frac{1}{\kappa} \varepsilon^{s} \mid\right\|_{\mathrm{UB}}^{2} \leq s \leq R^{\mathrm{P}}\left(\psi_{h}\right)+\frac{1}{4}\|\| \kappa e^{s}+\frac{1}{\kappa} \varepsilon^{s}\|\|_{\mathrm{UB}}^{2},\right.
$$

where the subscript UB denotes upper bound. 
Remark 1 The space $\widehat{\mathcal{W}}$ is obtained from $\mathcal{W}$ allowing time discontinuities at each time stage $t^{n}, n=1, \ldots, N-1$. Therefore, the primal and dual symmetric errors $e^{s}$ and $\varepsilon^{s}$ are in general discontinuous at these points in time. Moreover, the conditions given by equation (9) do not uniquely determine $e^{s}$ and $\varepsilon^{s}$ because $\mathcal{W} \subsetneq \widehat{\mathcal{W}}$.

Remark 2 For any $v \in \mathcal{W}$, the primal and adjoint residuals, defined in equations (3) and (7), may be rewritten as

$$
R^{P}(v)=\int_{0}^{T}\left[\left\langle f-\dot{u}_{h}, v\right\rangle-a\left(t ; u_{h}, v\right)\right] \mathrm{d} t=: \hat{R}^{P}(v)
$$

and

$$
R^{D}(v)=\int_{0}^{T}\left[\left\langle f^{\mathcal{O}}+\dot{\psi}_{h}, v\right\rangle-a\left(t ; v, \psi_{h}\right)\right] \mathrm{d} t=: \hat{R}^{D}(v) .
$$

by simply integrating by parts the term with the time derivative. This rearrangement of the residuals requires $v$ to be continuous and therefore it does not hold for $v \in \mathcal{W}$. That is, in general, for $v \in \widehat{\mathcal{W}}, R^{P}(v) \neq \hat{R}^{P}(v)$ and $R^{D}(v) \neq \hat{R}^{D}(v)$, see appendix $A$.

In practice, $e^{s}$ and $\varepsilon^{s}$ are chosen as the unique solution of the following residual equations: find $e^{s}$ and $\varepsilon^{s} \in \widehat{\mathcal{W}}$ such for any $v \in \widehat{\mathcal{W}}$
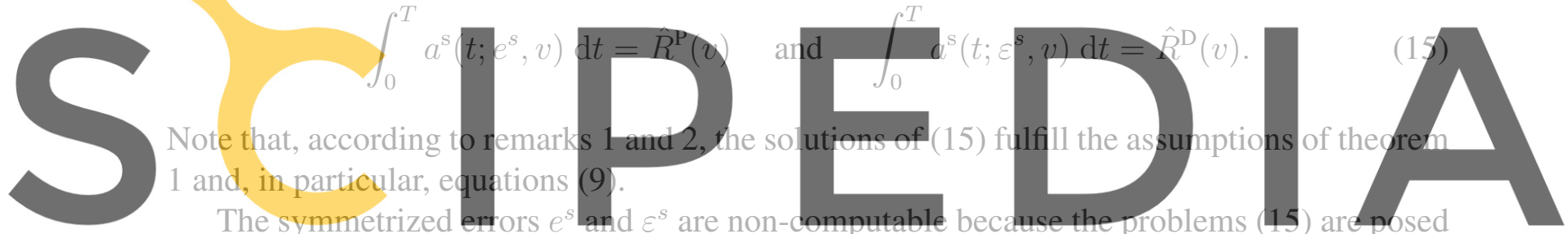

in infinite-dimensional spaces. With respect to the original error equations (3) and (7), equa-

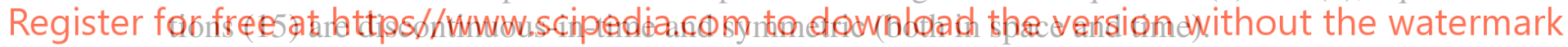

Next section is devoted to obtain computable upper bounds for the space-time norm $\||| \cdot||$ of the symmetrized errors $e^{s}$ and $\varepsilon^{s}$.

\section{Upper bounds for the space-time norm}

Consider the auxiliary function $z \in \widehat{\mathcal{W}}$ solution of

$$
\int_{0}^{T} a^{\mathrm{s}}(t ; z, v) \mathrm{d} t=\hat{R}^{*}(v) \quad \forall v \in \widehat{\mathcal{W}}
$$

where $\hat{R}^{*}(v)=\alpha \hat{R}^{\mathrm{P}}(v)+\beta \hat{R}^{\mathrm{D}}(v)$ for $\alpha, \beta \in \mathbb{R}$. Note that for $\alpha=1$ and $\beta=0$, then $\hat{R}^{*}(v)=\hat{R}^{\mathrm{P}}(v)$ and problem (16) is the residual problem for $e^{s}$. Therefore in this case $z=e^{s}$. Analogously, the choice of $\alpha=0$ and $\beta=1$, produces $\hat{R}^{*}(v)=\hat{R}^{\mathrm{D}}(v)$ and the residual problem for $\varepsilon^{s}$ is recovered yielding $z=\varepsilon^{s}$. In particular, $\alpha=\kappa$ and $\beta= \pm 1 / \kappa$ will be used later to obtain the required upper bounds for $\| \kappa e^{s} \pm 1 / \kappa \varepsilon^{s}||^{2}$.

The purpose of this section is to establish a procedure to compute upper bounds on $\|z \mid\|^{2}$. It is worth noting that the model problem under consideration, equation (16), is symmetric both in space and time and that it does not contain derivatives with respect to time. 
In order to come up with a computable upper bound of $\||z|\|^{2}$ the following four steps are considered. First, it is shown that $z \in \widehat{\mathcal{W}}$ may be computed solving $q+1$ independent steady diffusion-reaction problems in each slab $I_{n}$. Second, for every infinitely dimensional steady diffusion-reaction problem $(q+1$ in every time slab), the solution is decomposed in its projection into the finite element mesh $\mathcal{V}^{h}$ (which is computable) and the orthogonal complement (which is assessed with a standard error estimation technique). The problems characterizing the orthogonal complement are posed in the whole spatial domain $\Omega$. In the third step, a domain decomposition strategy is used to decompose the global problem into $n_{\mathrm{el}}$ independent (infinite dimensional) local problems defined in the elements of the mesh (triangles in our case), $n_{\mathrm{el}}$ being the number of elements of the spatial mesh. Finally, the fourth step uses a duality method to transform each local steady problem (posed over an infinite dimensional space) into a computable discrete problem yielding upper bounds of the solution.

\subsection{Time decomposition}

The first step to derive a computable expression for an upper bound of $\|z \mid\|^{2}$ is to decompose the global-in-time problem given by equation (16) into $q+1$ steady diffusion-reaction problems in each slab $I_{n}$

Using remark 2, for all $v \in \widehat{\mathcal{W}}$,

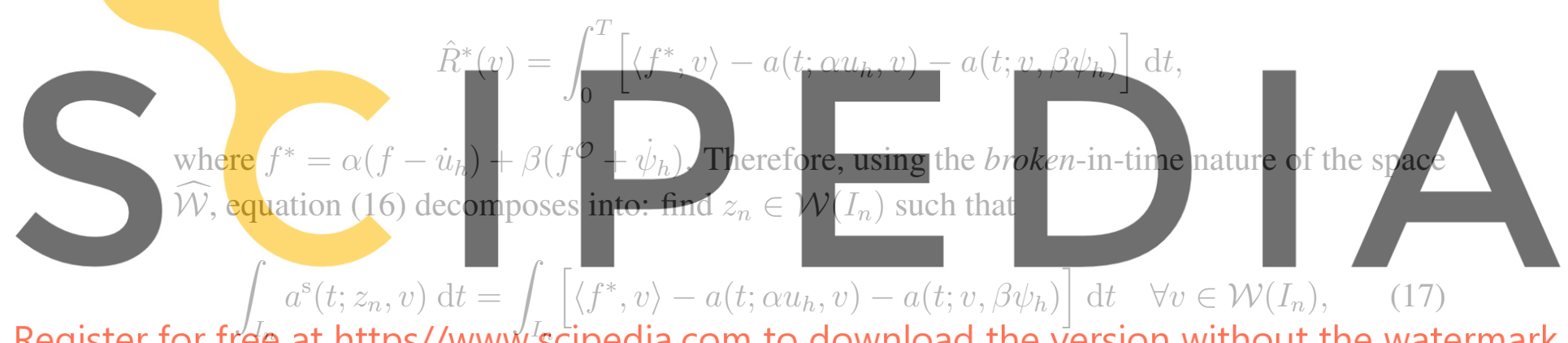

Register for free at https//www.scipedia.com to download the version without the watermark where

$$
\mathcal{V}\left(I_{n}\right):=\left\{v \in \mathcal{L}^{2}\left(I_{n} ; \mathcal{V}\right) \text { such that } v \in \mathcal{L}^{2}\left(I_{n} ; \mathcal{V}^{\prime}\right)\right\}
$$

Now, assume that $\nu(t), \boldsymbol{\alpha}(t)$ and $\sigma(t)$ are piecewise constant-in-time functions inside each time slab, that is

$$
\left.\nu(t)\right|_{I_{n}}=\nu_{n},\left.\quad \boldsymbol{\alpha}(t)\right|_{I_{n}}=\boldsymbol{\alpha}_{n} \quad \text { and }\left.\quad \sigma(t)\right|_{I_{n}}=\sigma_{n},
$$

for $\nu_{n}, \sigma_{n} \in \mathcal{L}^{\infty}(\Omega), \boldsymbol{\alpha}_{n} \in \mathcal{H}(\operatorname{div} ; \Omega)$ and $\boldsymbol{\nabla} \cdot \boldsymbol{\alpha}_{n}=0$. Working with piecewise constant-intime parameters has the advantage of avoiding the notational complexity introduced by more complex time dependencies. The proposed methodology is however more general in the sense that it is valid also for piecewise polynomial parameters $\nu(t), \boldsymbol{\alpha}(t)$ and $\sigma(t)$. In this case, however, computing $z_{n} \in \mathcal{W}\left(I_{n}\right)$ requires solving a larger number of steady diffusion-reaction problems in each slab $I_{n}$ (larger than $q+1$ ).

Under the assumption of piecewise constant-in-time parameters, the bilinear forms $a(t ; \cdot, \cdot)$ and $a^{\mathrm{s}}(t ; \cdot, \cdot)$ are also piecewise constant-in-time inside the time slabs, that is

$$
\left.a(t ; w, v)\right|_{I_{n}}=: a_{n}(w, v)=\int_{\Omega}\left[\nu_{n} \boldsymbol{\nabla} w \cdot \nabla v+\boldsymbol{\alpha}_{n} \cdot \nabla w v+\sigma_{n} w v\right] \mathrm{d} \Omega
$$


for $w, v \in \mathcal{L}^{2}(I ; \mathcal{V})$, and

$$
\left.a^{\mathrm{s}}(t ; w, v)\right|_{I_{n}}=: a_{n}^{\mathrm{s}}(w, v)=\int_{\Omega}\left[\nu_{n} \boldsymbol{\nabla} w \cdot \nabla v+\sigma_{n} w v\right] \mathrm{d} \Omega .
$$

The notation introduced above allows rewriting equation (17) as: find $z_{n} \in \mathcal{W}\left(I_{n}\right)$ such that

$$
\int_{I_{n}} a_{n}^{\mathrm{s}}\left(z_{n}, v\right) \mathrm{d} t=\int_{I_{n}}\left[\left\langle f^{*}, v\right\rangle-a_{n}\left(\alpha u_{h}, v\right)-a_{n}\left(v, \beta \psi_{h}\right)\right] \mathrm{d} t \quad \forall v \in \mathcal{W}\left(I_{n}\right) .
$$

The source terms are assumed to have a piecewise polynomial time-dependence, that is $\left.f\right|_{I_{n}}$ and $\left.f^{\mathcal{O}}\right|_{I_{n}}$ belong to $\mathbb{P}^{q}\left(I_{n} ; \mathcal{V}^{\prime}\right)$ and consequently $\left.f^{*}\right|_{I_{n}} \in \mathbb{P}^{q}\left(I_{n} ; \mathcal{V}^{\prime}\right)$. Recall that $u_{h}$ and $\psi_{h}$ belong to $\mathbb{P}^{q}\left(I_{n} ; \mathcal{V}\right)$ and therefore $z_{n} \in \mathbb{P}^{q}\left(I_{n} ; \mathcal{V}\right)$ and is such that

$$
a_{n}^{\mathrm{s}}\left(z_{n}, v\right)=\left\langle f^{*}, v\right\rangle-a_{n}\left(\alpha u_{h}, v\right)-a_{n}\left(v, \beta \psi_{h}\right) \quad \forall v \in \mathcal{W}\left(I_{n}\right) \quad \forall t \in I_{n} .
$$

The previous equation must be fulfilled for every $t \in I_{n}$. Nevertheless, due to the polynomial nature of $z_{n}$ it suffices to enforce it in $q+1$ time instants inside $I_{n}$.

Note that the time dependence of $z_{n}(t) \in \mathbb{P}^{q}\left(I_{n} ; \mathcal{V}\right)$ is uniquely characterized by giving $q+1$ space functions $z_{n i}, i=0,1, \ldots, q$, corresponding to the representation in a basis $N_{n i}(t)$, $i=0,1, \ldots, q$, of $\mathbb{P}^{q}\left(I_{n} ; \mathbb{R}\right)$, namely

$$
z_{n}(t)=\sum_{i=0}^{q} z_{n i} N_{n i}(t),
$$

where $z_{n i} \in \mathcal{V}$ are the space functions to be determined. Taking the basis $N_{n i}(t), i=$ $0,1, \ldots, q$, as the usual 1D finite element basis, see figure 1 , the degrees of freedom $z_{n i}$ correspond to the values of $z_{n}$ at the intermediate times $t^{n-1+i / q}:=t^{n-1}+i\left(t^{n}-t^{n-1}\right) / q$, that is $z_{n i}=z_{n}\left(t^{n-1+i / q}\right)$, for $i=0,1, \ldots, q$. For simplicity, $t^{n-1+i / q}$ will be denoted by $t_{n i}$ in the following.
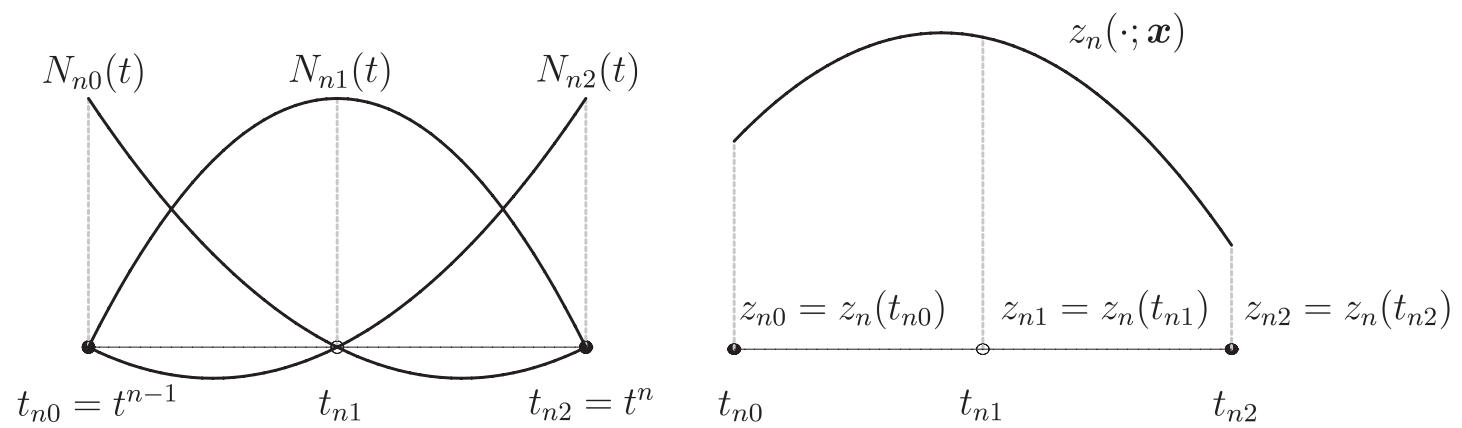

Figure 1: Representation for $q=2$ of the time dependency of the function $z_{n}$.

In this case, $z_{n i} \in \mathcal{V}$ is the solution of the steady diffusion-reaction problem

$$
a_{n}^{\mathrm{s}}\left(z_{n i}, v\right)=\left\langle f^{*}\left(t_{n i}\right), v\right\rangle-a_{n}\left(\alpha u_{h}\left(t_{n i}\right), v\right)-a_{n}\left(v, \beta \psi_{h}\left(t_{n i}\right)\right) \quad \forall v \in \mathcal{V} .
$$

Thus, the solution of equation (16), $z \in \widehat{\mathcal{W}}$, is computed solving $q+1$ independent steady diffusion-reaction problems in each time slab $I_{n}$. Once the functions $z_{n i} \in \mathcal{V}$ are obtained solving (21), the time-dependent function $z_{n}(t) \in \mathbb{P}^{q}\left(I_{n} ; \mathcal{V}\right)$ is directly recovered using equation (20). The assembly of these solutions associated with every time slab makes $z \in \widehat{\mathcal{W}}$ a piecewise polynomial function in time, discontinuous at $t=t^{n}$ for $n=1, \ldots, N-1$ (recall that $\left.z\right|_{I_{n}}=z_{n}$ ). 
Remark 3 As already mentioned, the assumption that $\nu(t), \boldsymbol{\alpha}(t)$ and $\sigma(t)$ are piecewise constantin-time functions inside each time slab, and also that $\left.f^{*}\right|_{I_{n}} \in \mathbb{P}^{q}\left(I_{n} ; \mathcal{V}^{\prime}\right)$ may be relaxed. In fact, if $\left.\nu\right|_{I_{n}},\left.\boldsymbol{\alpha}\right|_{I_{n}},\left.\sigma\right|_{I_{n}}$ and $\left.f^{*}\right|_{I_{n}}$ are polynomial functions inside $I_{n}$, then a larger degree of the polynomials representing the time dependence of the solution $z_{n}$, say $\tilde{q}$, can be selected such that $z_{n}$ belongs to $\mathbb{P}^{\tilde{q}}\left(I_{n} ; \mathcal{V}\right)$. Then, $z_{n}$ must be computed solving $\tilde{q}+1$ independent steady diffusion-reaction problems.

\subsection{Enforcing orthogonality}

The solution $z_{n i}$ of the steady diffusion-reaction (21) and therefore the norm $\|\mid\|^{2} \|^{2}$ to be used in the bounds are not computable because $\mathcal{V}$ is infinite-dimensional. It is however possible to derive a computable upper bound for $\|\mid z\|^{2}$ using a domain decomposition technique and, in every local problem, a complementary energy approach. The idea is to use the error estimation strategy proposed in [18] to each steady reaction-diffusion problem (21) as in [15]. However, these techniques may only be applied if the r.h.s. of the residual equation, in this case equation (21), vanishes for every $v \in \mathcal{V}^{h}$ (this is referred as the orthogonality property) $[2,8,11,16]$. This orthogonality condition is needed to properly produce the domain decomposition strategies and the equilibration of the local problems.

As already noted, recall (4) and (8), the orthogonality is not fulfilled. That is, in general for $v \in \mathcal{V}^{h}$

$$
\left\langle f^{*}\left(t_{n i}\right), v\right\rangle-a_{n}\left(\alpha u_{h}\left(t_{n i}\right), v\right)-a_{n}\left(v, \beta \psi_{h}\left(t_{n i}\right)\right) \neq 0 .
$$

This problem may be circumvented decomposing $z_{n i}$ into

$$
z_{n i}=z_{n i}^{h}+z_{n i}^{\perp}
$$

where $z_{n i}^{h} \in \mathcal{V}^{h}$ is such that

$$
a_{n}^{\mathrm{s}}\left(z_{n i}^{h}, v\right)=\left\langle f^{*}\left(t_{n i}\right), v\right\rangle-a_{n}\left(\alpha u_{h}\left(t_{n i}\right), v\right)-a_{n}\left(v, \beta \psi_{h}\left(t_{n i}\right)\right) \quad \forall v \in \mathcal{V}^{h} .
$$

Note that the $z_{n i}^{h}$ is the projection of $z_{n i}$ into $\mathcal{V}^{h}$. Thus, from (21) the orthogonal complement $z_{n i}^{\perp} \in \mathcal{V}$ is the solution of the residual equation

$$
a_{n}^{\mathrm{s}}\left(z_{n i}^{\perp}, v\right)=\left\langle f^{*}\left(t_{n i}\right), v\right\rangle-a_{n}\left(\alpha u_{h}\left(t_{n i}\right), v\right)-a_{n}\left(v, \beta \psi_{h}\left(t_{n i}\right)\right)-a_{n}^{\mathrm{s}}\left(z_{n i}^{h}, v\right) \quad \forall v \in \mathcal{V} .
$$

This decomposition precludes the problem associated with the lack of orthogonality because $z_{n i}^{h}$ is computable and equation (23) for $z_{n i}^{\perp}$, is such that the r.h.s. fulfills the orthogonality condition. That is, the r.h.s. of (23) vanishes for every $v \in \mathcal{V}^{h}$. Therefore, a computable bound for the norm of $z_{n i}^{\perp}$ is obtained after a domain decomposition technique.

This orthogonalization strategy is used in [7, 16] in a different context (assessment of the pollution). A similar approach is used also in [19] to recover strict bounds for the energy.

Summarizing, the upper bound for $\||z|\|^{2}$ requires computing first $z_{n i}^{h} \in \mathcal{V}^{h}$ and then applying the error estimation technique proposed in [18] to approximate $z_{n i}^{\perp}$. This has to be performed for every time $t_{n i}\left(q+1\right.$ times in each time slab $\left.I_{n}\right)$.

\subsection{Domain decomposition and complementary energy approach}

Now, the standard complementary energy approach is applied to obtain estimates for $z_{n i}^{\perp}$ given by equation (23) following the ideas introduced in $[17,18,15]$. Note that this is possible because orthogonality has been enforced as described in the previous section. 
The basic idea is to relax the problem of finding $z_{n i}^{\perp} \in \mathcal{V}$ fulfilling equation (23). The relaxed problem consists in obtaining a pair of dual estimates $\hat{\boldsymbol{p}}_{n i} \in\left[\mathcal{L}^{2}(\Omega)\right]^{\mathrm{n}_{\mathrm{sd}}}$ and $\hat{r}_{n i} \in \mathcal{L}^{2}(\Omega)$ such that

$$
\int_{\Omega}\left[\nu_{n} \hat{\boldsymbol{p}}_{n i} \cdot \boldsymbol{\nabla} v+\sigma_{n} \hat{r}_{n i} v\right] \mathrm{d} \Omega=a_{n}^{\mathrm{s}}\left(z_{n i}^{\perp}, v\right) \quad \forall v \in \mathcal{V} .
$$

The estimates for $\hat{\boldsymbol{p}}_{n i}$ and $\hat{r}_{n i}$ are taken in an elementwise-polynomial space of degree $r$, namely

$$
\widehat{\mathbb{P}}^{r}(\Omega):=\left\{v \in \mathcal{L}^{2}(\Omega),\left.v\right|_{\Omega_{k}} \in \mathbb{P}^{r}\left(\Omega_{k}\right)\right\},
$$

i.e. $\hat{r}_{n i} \in \widehat{\mathbb{P}}^{r}(\Omega)$ and $\hat{\boldsymbol{p}}_{n i} \in\left[\widehat{\mathbb{P}}^{r}(\Omega)\right]^{2}$, where $\Omega_{k}$ for $k=1,2, \ldots, n_{\mathrm{el}}$ are the elements of the mesh. The fact that the data fields $u_{0}, u_{T}^{\mathcal{O}}, f(t)$ and $f^{\mathcal{O}}(t)$ are assumed to be piecewise polynomials both in space (element by element) and time (in each time slab) guarantees that for $r$ large enough a pair of dual estimates fulfilling equation (24) may be found in $\widehat{\mathbb{P}}^{r}(\Omega)$. This results in a discrete solvable problem, see $[17,18]$.

Remark 4 The dual estimates $\hat{\boldsymbol{p}}_{n i}$ and $\hat{r}_{n i}$ are defined over the whole domain $\Omega$. Nevertheless, their computation can be decoupled locally by selecting appropriate parameterizations. In practice, the equilibration procedure yielding equilibrated fluxes is local and the a priori global problem (24) is split into local dual problems in the elements of the mesh.

\subsection{Computation of an upper bound}

Finally, the estimates obtained from (24) are combined with the projections computed from (22) to build up an upper bound for $\|z\| \mid \|$. This is stated in the following theorem.

Theorem 2 Let $\hat{\boldsymbol{p}}_{n i} \in\left[\widehat{\mathbb{P}}^{r}(\Omega)\right]^{\mathrm{n}_{\mathrm{sd}}}$ and $\hat{r}_{n i} \in \widehat{\mathbb{P}}^{r}(\Omega)$ be the dual estimates fulfilling equation (24) for every $t_{n i}, n=1,2, \ldots, N, i=0,1, \ldots, q$. The time-dependent estimates are readily recovered in every time slab $I_{n}$ :

$$
\hat{\boldsymbol{p}}_{n}=\sum_{i=0}^{q} \hat{\boldsymbol{p}}_{n i} N_{n i}(t) \text { and } \hat{r}_{n}=\sum_{i=0}^{q} \hat{r}_{n i} N_{n i}(t) .
$$

Analogously, let $z_{n i}^{h} \in \mathcal{V}^{h}$ be the solutions of (22) and

$$
z_{n}^{h}=\sum_{i=0}^{q} z_{n i}^{h} N_{n i}(t)
$$

Then, an upper bound for the space-time norm of the solution $z$ of (16) is computed as

$$
\|z \mid\|^{2} \leq \sum_{n=1}^{N} \int_{I_{n}} \int_{\Omega}\left[\nu_{n}\left(\hat{\boldsymbol{p}}_{n}+\boldsymbol{\nabla} z_{n}^{h}\right) \cdot\left(\hat{\boldsymbol{p}}_{n}+\boldsymbol{\nabla} z_{n}^{h}\right)+\sigma_{n}\left(\hat{r}_{n}+z_{n}^{h}\right)^{2}\right] \mathrm{d} \Omega \mathrm{d} t .
$$

Proof. Let $z_{n}^{\perp}=\sum_{i=0}^{q} z_{n i}^{\perp} N_{n i}(t)$, where $z_{n i}^{\perp}$ are the solutions of (23). Then, from equation (24) and using equation (23), the dual estimates $\hat{\boldsymbol{p}}_{n}$ and $r_{n}$ verify for $i=0, \ldots, q$

$$
\begin{aligned}
& \int_{\Omega}\left[\nu_{n} \hat{\boldsymbol{p}}_{n}\left(t_{n i}\right) \cdot \nabla v+\sigma_{n} \hat{r}_{n}\left(t_{n i}\right) v\right] \mathrm{d} \Omega \\
& \quad=\left\langle f^{*}\left(t_{n i}\right), v\right\rangle-a_{n}\left(\alpha u_{h}\left(t_{n i}\right), v\right)-a_{n}\left(v, \beta \psi_{h}\left(t_{n i}\right)\right)-a_{n}^{\mathrm{s}}\left(z_{n}^{h}\left(t_{n i}\right), v\right) \quad \forall v \in \mathcal{V},
\end{aligned}
$$


since $\hat{\boldsymbol{p}}_{n i}=\hat{\boldsymbol{p}}_{n}\left(t_{n i}\right), r_{n i}=\hat{r}_{n}\left(t_{n i}\right)$ and $z_{n}^{h}\left(t_{n i}\right)=z_{n i}^{h}$. Moreover, since all the time-dependent functions appearing in the previous equation are polynomials of degree $q$, the previous equation is verified for every $t \in I_{n}$, that is,

$$
\begin{aligned}
& \int_{\Omega}\left[\nu_{n} \hat{\boldsymbol{p}}_{n}(t)\right.\left.\cdot \boldsymbol{\nabla} v+\sigma_{n} \hat{r}_{n}(t) v\right] \mathrm{d} \Omega+a_{n}^{\mathrm{s}}\left(z_{n}^{h}(t), v\right) \\
&=\left\langle f^{*}(t), v\right\rangle-a_{n}\left(\alpha u_{h}(t), v\right)-a_{n}\left(v, \beta \psi_{h}(t)\right) \quad \forall v \in \mathcal{V} \forall t \in I_{n} .
\end{aligned}
$$

Integrating from $t=t^{n-1}=t_{n 0}$ to $t=t^{n}=t_{n q}$, and expanding the term $a_{n}^{\mathrm{s}}\left(z_{n}^{h}(t), v\right)$ using equation (18) yields

$$
\begin{aligned}
& \int_{I_{n}} \int_{\Omega}\left[\nu_{n}\left(\hat{\boldsymbol{p}}_{n}+\nabla z_{n}^{h}\right) \cdot \nabla v+\sigma_{n}\left(\hat{r}_{n}+z_{n}^{h}\right) v\right] \mathrm{d} \Omega \mathrm{d} t \\
& \quad=\int_{I_{n}}\left[\left\langle f^{*}, v\right\rangle-a_{n}\left(\alpha u_{h}, v\right)-a_{n}\left(v, \beta \psi_{h}\right)\right] \mathrm{d} t=\int_{I_{n}} a_{n}^{\mathrm{s}}\left(z_{n}, v\right) \mathrm{d} t \quad \forall v \in \mathcal{W}\left(I_{n}\right),
\end{aligned}
$$

where in the last equality, equation (19) has been used. In particular, taking $v=z_{n} \in \mathcal{W}\left(I_{n}\right)$ in the previous equation yields:

$$
\int_{I_{n}} \int_{\Omega}\left[\nu_{n}\left(\hat{\boldsymbol{p}}_{n}+\nabla z_{n}^{h}\right) \cdot \nabla z_{n}+\sigma_{n}\left(\hat{r}_{n}+z_{n}^{h}\right) z_{n}\right] \mathrm{d} \Omega \mathrm{d} t=\int_{I_{n}} a_{n}^{\mathrm{s}}\left(z_{n}, z_{n}\right) \mathrm{d} t .
$$

At this point, the previous equality along with an elementary algebraic manipulations reveal that

$$
\int_{I_{n}} a_{n}^{\mathrm{s}}\left(z_{n}, z_{n}\right) \mathrm{d} t \leq \int_{I_{n}} \int_{\Omega}\left[\nu_{n}\left(\hat{\boldsymbol{p}}_{n}+\boldsymbol{\nabla} z_{n}^{h}\right) \cdot\left(\hat{\boldsymbol{p}}_{n}+\boldsymbol{\nabla} z_{n}^{h}\right)+\sigma_{n}\left(\hat{r}_{n}+z_{n}^{h}\right)^{2}\right] \mathrm{d} \Omega \mathrm{d} t .
$$

Indeed, the result is obtained using the obvious inequality

$$
\int_{I_{n}} \int_{\Omega}\left[\nu_{n}\left(\hat{\boldsymbol{p}}_{n}+\boldsymbol{\nabla} z_{n}^{h}-\boldsymbol{\nabla} z_{n}\right) \cdot\left(\hat{\boldsymbol{p}}_{n}+\boldsymbol{\nabla} z_{n}^{h}-\boldsymbol{\nabla} z_{n}\right)+\sigma_{n}\left(\hat{r}_{n}+z_{n}^{h}-z_{n}\right)^{2}\right] \mathrm{d} \Omega \mathrm{d} t \geq 0
$$

along with the algebraic manipulation

$$
\begin{aligned}
& \int_{I_{n}} \int_{\Omega}\left[\nu_{n}\left(\hat{\boldsymbol{p}}_{n}+\nabla z_{n}^{h}-\boldsymbol{\nabla} z_{n}\right) \cdot\left(\hat{\boldsymbol{p}}_{n}+\boldsymbol{\nabla} z_{n}^{h}-\boldsymbol{\nabla} z_{n}\right)+\sigma_{n}\left(\hat{r}_{n}+z_{n}^{h}-z_{n}\right)^{2}\right] \mathrm{d} \Omega \mathrm{d} t \\
& =\int_{I_{n}} \int_{\Omega}\left[\nu_{n}\left(\hat{\boldsymbol{p}}_{n}+\boldsymbol{\nabla} z_{n}^{h}\right) \cdot\left(\hat{\boldsymbol{p}}_{n}+\boldsymbol{\nabla} z_{n}^{h}\right)+\sigma_{n}\left(\hat{r}_{n}+z_{n}^{h}\right)^{2}\right] \mathrm{d} \Omega \mathrm{d} t \\
& \quad+\int_{I_{n}} \int_{\Omega}\left[\nu_{n} \boldsymbol{\nabla} z_{n} \cdot \boldsymbol{\nabla} z_{n}+\sigma_{n}\left(z_{n}\right)^{2}\right] \mathrm{d} \Omega \mathrm{d} t \\
& \quad-2 \int_{I_{n}} \int_{\Omega}\left[\nu_{n}\left(\hat{\boldsymbol{p}}_{n}+\boldsymbol{\nabla} z_{n}^{h}\right) \cdot \boldsymbol{\nabla} z_{n}+\sigma_{n}\left(\hat{r}_{n}+z_{n}^{h}\right) z_{n}\right] \mathrm{d} \Omega \mathrm{d} t \\
& =\int_{I_{n}} \int_{\Omega}\left[\nu_{n}\left(\hat{\boldsymbol{p}}_{n}+\boldsymbol{\nabla} z_{n}^{h}\right) \cdot\left(\hat{\boldsymbol{p}}_{n}+\boldsymbol{\nabla} z_{n}^{h}\right)+\sigma_{n}\left(\hat{r}_{n}+z_{n}^{h}\right)^{2}\right] \mathrm{d} \Omega \mathrm{d} t \\
& \quad+\int_{I_{n}} a_{n}^{\mathrm{s}}\left(z_{n}, z_{n}\right) \mathrm{d} t-2 \int_{I_{n}} a_{n}^{\mathrm{s}}\left(z_{n}, z_{n}\right) \mathrm{d} t \\
& =\int_{I_{n}} \int_{\Omega}\left[\nu_{n}\left(\hat{\boldsymbol{p}}_{n}+\boldsymbol{\nabla} z_{n}^{h}\right) \cdot\left(\hat{\boldsymbol{p}}_{n}+\boldsymbol{\nabla} z_{n}^{h}\right)+\sigma_{n}\left(\hat{r}_{n}+z_{n}^{h}\right)^{2}\right] \mathrm{d} \Omega \mathrm{d} t-\int_{I_{n}} a_{n}^{\mathrm{s}}\left(z_{n}, z_{n}\right) \mathrm{d} t,
\end{aligned}
$$


where both equations (27) and (18) have been used.

Finally, using the inequality given by equation (28)

$$
\begin{aligned}
\|z\|^{2} & =\int_{0}^{T}\|z\|^{2} \mathrm{~d} t=\int_{0}^{T} a^{\mathrm{s}}(t ; z, z) \mathrm{d} t \\
& =\sum_{n=1}^{N} \int_{I_{n}} a^{\mathrm{s}}(t ; z, z) \mathrm{d} t=\sum_{n=1}^{N} \int_{I_{n}} a_{n}^{\mathrm{s}}\left(z_{n}, z_{n}\right) \mathrm{d} t \\
& \leq \sum_{n=1}^{N} \int_{I_{n}} \int_{\Omega}\left[\nu_{n}\left(\hat{\boldsymbol{p}}_{n}+\nabla z_{n}^{h}\right) \cdot\left(\hat{\boldsymbol{p}}_{n}+\nabla z_{n}^{h}\right)+\sigma_{n}\left(\hat{r}_{n}+z_{n}^{h}\right)^{2}\right] \mathrm{d} \Omega \mathrm{d} t,
\end{aligned}
$$

concluding the proof.

\section{Bounds for the output of interest $L^{\mathcal{O}}(u)$ : an algorithmic summary}

According to theorem 1 the upper and lower bounds of $s$, and hence of $L^{\mathcal{O}}(u)$, are available once the upper bounds of the energy norm $\||z| \mid$ are obtained for the two combinations $(\alpha, \beta)=$ $(\kappa, 1 / \kappa)$ and $(\alpha, \beta)=(\kappa,-1 / \kappa)$. The general strategy to obtain these upper bounds is devised in the previous section. As already mentioned, due to the linearity of the problem, obtaining the estimates for these two values of $\left\||z \||\right.$ is equivalent to obtain the estimates for $z=e^{s}$ and $z=\varepsilon^{s}$, that is for the two combinations $(\alpha, \beta)=(1,0)$ and $(\alpha, \beta)=(0,1)$.

The following description of the bound algorithm differs from the description given in part I of this work [15] because here the algorithm is designed to parallelize the computation of the estimates in each time slab. However, if memory requirements are critical, the same strategy proposed in [15] can be implemented. That is, only the primal solution must be stored. The adjoint one is computed (but not stored) step by step in each time slab.

The main steps of the procedure to compute bounds for $L^{\mathcal{O}}(u)$ are the following:

1. Compute and store the continuous primal and dual solutions $u_{h}$ and $\psi_{h}$ respectively (for instance, computing the $\mathrm{cG}(p) \mathrm{dG}(q)$ approximations and smoothing out the time discontinuities).

2. For each time slab $I_{n}$ do (this step is independent for each slab and can be easily parallelized):

2.2. For each subtime $t_{n i}, i=0, \ldots, q$ do:

2.2.1. Compute the primal and adjoint projections $e_{n i}^{s, h}$ and $\varepsilon_{n i}^{s, h} \in \mathcal{V}^{h}$ solution of:

$$
\begin{array}{cc}
a_{n}^{\mathrm{s}}\left(e_{n i}^{s, h}, v\right)=\left\langle f\left(t_{n i}\right)-\dot{u}_{h}\left(t_{n i}\right), v\right\rangle-a_{n}\left(u_{h}\left(t_{n i}\right), v\right) & \forall v \in \mathcal{V}^{h}, \\
a_{n}^{\mathrm{s}}\left(\varepsilon_{n i}^{s, h}, v\right)=\left\langle f^{\mathcal{O}}\left(t_{n i}\right)+\dot{\psi}_{h}\left(t_{n i}\right), v\right\rangle-a_{n}\left(v, \psi_{h}\left(t_{n i}\right)\right) & \forall v \in \mathcal{V}^{h} .
\end{array}
$$

2.2.2. Compute the primal and adjoint dual estimates $\hat{\boldsymbol{p}}_{n i}^{P}, \hat{\boldsymbol{p}}_{n i}^{D} \in\left[\widehat{\mathbb{P}}^{r}(\Omega)\right]^{\mathrm{n}_{\mathrm{sd}}}$ and $\hat{r}_{n i}^{P}$, $\hat{r}_{n i}^{D} \in \widehat{\mathbb{P}}^{r}(\Omega)$ such that for all $v \in \mathcal{V}$ :

$$
\begin{aligned}
& \int_{\Omega}\left[\nu_{n} \hat{\boldsymbol{p}}_{n i}^{P} \cdot \nabla v+\sigma_{n} \hat{r}_{n i}^{P} v\right] \mathrm{d} \Omega \\
& \quad=\left\langle f\left(t_{n i}\right)-\dot{u}_{h}\left(t_{n i}\right), v\right\rangle-a_{n}\left(u_{h}\left(t_{n i}\right), v\right)-a_{n}^{\mathrm{s}}\left(e_{n i}^{s, h}, v\right)
\end{aligned}
$$




$$
\begin{aligned}
\int_{\Omega}\left[\nu_{n} \hat{\boldsymbol{p}}_{n i}^{D} \cdot \nabla v+\sigma_{n} \hat{r}_{n i}^{D} v\right] \mathrm{d} \Omega \\
\quad=\left\langle f^{\mathcal{O}}\left(t_{n i}\right)+\dot{\psi}_{h}\left(t_{n i}\right), v\right\rangle-a_{n}\left(v, \psi_{h}\left(t_{n i}\right)\right)-a_{n}^{\mathrm{s}}\left(\varepsilon_{n i}^{s, h}, v\right) .
\end{aligned}
$$

2.3 For each element, recover the time-dependent projections in the time slab $I_{n}$

$$
e_{n k}^{s, h}(t)=\left.\sum_{i=0}^{q} e_{n i}^{s, h}\right|_{\Omega_{k}} N_{n i}(t) \text { and } \varepsilon_{n k}^{s, h}(t)=\left.\sum_{i=0}^{q} \varepsilon_{n i}^{s, h}\right|_{\Omega_{k}} N_{n i}(t),
$$

and the dual time-dependent estimates

$$
\begin{aligned}
& \hat{\boldsymbol{p}}_{n k}^{P}(t)=\left.\sum_{i=0}^{q} \hat{\boldsymbol{p}}_{n i}^{P}\right|_{\Omega_{k}} N_{n i}(t) \text { and } \hat{r}_{n k}^{P}(t)=\left.\sum_{i=0}^{q} \hat{r}_{n i}^{P}\right|_{\Omega_{k}} N_{n i}(t), \\
& \hat{\boldsymbol{p}}_{n k}^{D}(t)=\left.\sum_{i=0}^{q} \hat{\boldsymbol{p}}_{n i}^{D}\right|_{\Omega_{k}} N_{n i}(t) \text { and } \hat{r}_{n k}^{D}(t)=\left.\sum_{i=0}^{q} \hat{r}_{n i}^{D}\right|_{\Omega_{k}} N_{n i}(t) .
\end{aligned}
$$

2.4 Compute and store the three scalar quantities

$$
\begin{array}{r}
\eta_{n}^{P}:=\sum_{k=1}^{n_{\mathrm{el}}} \eta_{n k}^{P}=\sum_{k=1}^{n_{\mathrm{el}}} \int_{I_{n}} \int_{\Omega_{k}}\left[\nu_{n}\left(\hat{\boldsymbol{p}}_{n k}^{P}+\nabla e_{n k}^{s, h}\right)^{2}+\sigma_{n}\left(\hat{r}_{n k}^{P}+e_{n k}^{s, h}\right)^{2}\right] \mathrm{d} \Omega \mathrm{d} t, \\
\eta_{n}^{D}:=\sum_{k=1}^{n_{\mathrm{el}}} \eta_{n k}^{D}=\sum_{k=1}^{n_{\mathrm{el}}} \int_{I_{n}} \int_{\Omega_{k}}\left[\nu_{n}\left(\hat{\boldsymbol{p}}_{n k}^{D}+\nabla \varepsilon_{n k}^{s, h}\right)^{2}+\sigma_{n}\left(\hat{r}_{n k}^{D}+\varepsilon_{n k}^{s, h}\right)^{2}\right] \mathrm{d} \Omega \mathrm{d} t, \\
\eta_{n}^{P D}:=\sum_{k=1}^{n_{\mathrm{el}}} \eta_{n k}^{P D}=\sum_{k=1}^{n_{\mathrm{el}}} \int_{I_{n}} \int_{\Omega_{k}}\left[\nu_{n}\left(\hat{\boldsymbol{p}}_{n k}^{P}+\boldsymbol{\nabla} e_{n k}^{s, h}\right) \cdot\left(\hat{\boldsymbol{p}}_{n k}^{D}+\nabla \varepsilon_{n k}^{s, h}\right)\right. \\
\left.+\sigma_{n}\left(\hat{r}_{n k}^{P}+e_{n k}^{s, h}\right)\left(\hat{r}_{n k}^{D}+\varepsilon_{n k}^{s, h}\right)\right] \mathrm{d} \Omega \mathrm{d} t .
\end{array}
$$

3. Compute the global quantities

$$
\eta^{P}=\left(\sum_{i=1}^{N} \eta_{n}^{P}\right)^{\frac{1}{2}}, \eta^{D}=\left(\sum_{i=1}^{N} \eta_{n}^{D}\right)^{\frac{1}{2}}, \eta^{P D}=\sum_{i=1}^{N} \eta_{n}^{P D}
$$

and recover the bounds for the output $s^{-} \leq L^{\mathcal{O}}(u) \leq s^{+}$where

$$
s^{-}:=L^{\mathcal{O}}\left(u_{h}\right)+R^{\mathrm{P}}\left(\psi_{h}\right)-\frac{1}{2} \eta^{P} \eta^{D}+\frac{1}{2} \eta^{P D}
$$

and

$$
s^{+}=L^{\mathcal{O}}\left(u_{h}\right)+R^{\mathrm{P}}\left(\psi_{h}\right)+\frac{1}{2} \eta^{P} \eta^{D}+\frac{1}{2} \eta^{P D} .
$$

Remark 5 The final expression for the bounds of the output $L^{\mathcal{O}}(u)$ are recovered by means of the following considerations. First theorem 1 states that in order to obtain bounds for the error in the output $s$ it is sufficient to obtain upper bounds for the quantities $\left\|\kappa e^{s} \pm \frac{1}{\kappa} \varepsilon^{s}\right\| \|_{U B}^{2}$, see equation (14). In order to compute the upper bounds for the space-time norm, the procedure 
detailed in section 5 is considered for $z=\kappa e^{s} \pm \frac{1}{\kappa} \varepsilon^{s}$. Then, from theorem 2, the following upper bounds are obtained:

$$
\begin{aligned}
\left\|\kappa e^{s} \pm \frac{1}{\kappa} \varepsilon^{s}\right\| \|_{U B}^{2}=\sum_{i=1}^{N} \int_{I_{n}} \int_{\Omega}[ & \nu_{n}\left(\kappa\left(\hat{\boldsymbol{p}}_{n}^{P}+\nabla e_{n}^{s, h}\right) \pm \frac{1}{\kappa}\left(\hat{\boldsymbol{p}}_{n}^{D}+\nabla \varepsilon_{n}^{s, h}\right)\right)^{2} \\
+ & \left.\sigma_{n}\left(\kappa\left(\hat{r}_{n}^{P}+e_{n}^{s, h}\right) \pm \frac{1}{\kappa}\left(\hat{r}_{n}^{D}+\varepsilon_{n}^{s, h}\right)\right)^{2}\right] \mathrm{d} \Omega \mathrm{d} t .
\end{aligned}
$$

Finally the given expressions for the bounds are obtained taking $\kappa^{2}=\eta^{D} / \eta^{P}$ and rearranging terms.

\section{Numerical examples}

The numerical tests presented in this section are the same as in the first part of the paper [15] but with the numerical tools introduced in this second part. These examples are used to demonstrate the ability of the presented approach to account also for the error associated with the time discretization and to point out the difficulties in recovering the predicted a priori convergence rates. Moreover, $h$-adaptivity does no longer suffice here to control the error and $\Delta t$ has to be adapted also to reduce the error assessed with the presented strategy (including the time error). The notation used here is therefore the same as in [15] and it is briefly recalled. The upper and lower bounds for $L^{\mathcal{O}}(u)$ introduced above are denoted by $s^{-}$and $s^{+}$. The bound average, $s^{\text {ave }}:=\left(s^{+}+s^{-}\right) / 2$, is taken as a new approximation of the quantity of interest and the half bound gap, $\Delta=\left(s^{+}-s^{-}\right) / 2$, is seen as an error indicator. Note that,

$$
\left|L^{\mathcal{O}}(u)-s^{\text {ave }}\right| \leq \Delta
$$

that is, $\Delta$ measures the absolute error of the approximation $s^{\text {ave }}$ with respect to the exact value $L^{\mathcal{O}}(u)$. Since the exact solution of the problems is not known, the relative counterpart of the half bound gap, $\Delta_{\text {rel }}=\Delta / s^{\text {ave }}$, is also used in the presentation.

The meshes are adapted to reduce the half bound gap, $\Delta$. In the examples a simple adaptive strategy is used based on the decomposition of $\Delta$ into local positive contributions from the elements:

$$
\Delta=\sum_{k=1}^{n_{\mathrm{el}}} \Delta_{k}
$$

where the element contribution to the bound gap $\Delta_{k}$ is

$$
\Delta_{k}:=\sum_{i=1}^{N}\left[\frac{1}{4} \kappa^{2} \eta_{n k}^{P}+\frac{1}{4 \kappa^{2}} \eta_{n k}^{D}\right] .
$$

The validity of this decomposition is discussed in [15]. The remeshing strategy (with respect to space) consists in subdividing, at each step of the adaptive procedure, the elements with the larger values of $\Delta_{k}$. 


\subsection{Example 1: uniformly forced square domain}

The transient pure diffusion equation $(\nu=1, \sigma=0, \boldsymbol{\alpha}=0)$ is solved in the squared domain $\Omega=[0,1] \times[0,1]$ and for a final time $T=0.1$. A constant source term $f(t)=\sqrt{10}$ and homogeneous Dirichlet boundary conditions and initial condition $\left(u_{0}=0\right)$ are considered.

The quantity of interest is an average of the space-time solution

$$
L^{\mathcal{O}}(u)=\int_{0}^{T} \int_{\Omega} \sqrt{10} u(x, y, t) \mathrm{d} \Omega \mathrm{d} t,
$$

that is $f^{\mathcal{O}}=\sqrt{10}$ and $u_{T}^{\mathcal{O}}=0$ in equation (5). The solution $\psi$ of the adjoint problem is in this case such that $u(t)=\psi(T-t)$.

Two spatial discretizations are used in this test: linear and quadratic triangular elements in space, $p=1$ and $p=2$. In the computation of the hybrid fluxes, the equilibrated normal fluxes along the edges of the elements are linear, both for $p=1$ and $p=2$. The local approximation to $\hat{Z}_{n j k}$ and its fluxes in the interior of the elements, $\hat{\boldsymbol{P}}_{n j k} \in\left[\mathbb{P}^{r}\left(\Omega_{k}\right)\right]^{\mathrm{n}_{\mathrm{sd}}}$ and $\hat{R}_{n j k} \in \mathbb{P}^{r}\left(\Omega_{k}\right)$ are fourth order polynomials, i.e. $r=4$.

The convergence of the bounds is analyzed for a uniform mesh refinement in a series of structured meshes. The initial mesh is composed by 8 triangular elements (half squares) and in each refinement step every triangle is divided in four similar triangles. The study is done using different strategies to determine the time step: in each refinement step the time step is kept constant $\Delta t_{n}=\Delta t$ (all the time slabs $I_{n}$ have the same size) but different strategies are used to modify the time step $\Delta t$ as the meshes are refined uniformly.

The results for linear elements $(p=1)$ are shown in figure 2 for 3 cases: 1$)$ constant time step $\Delta t=0.05(N=20)$ during all the mesh refinement procedure, 2$)$ varying the time step as $\Delta t \propto \sqrt{t}$ and 3) varying the time step as $\Delta t \propto h$. The optimal finite element convergence rate

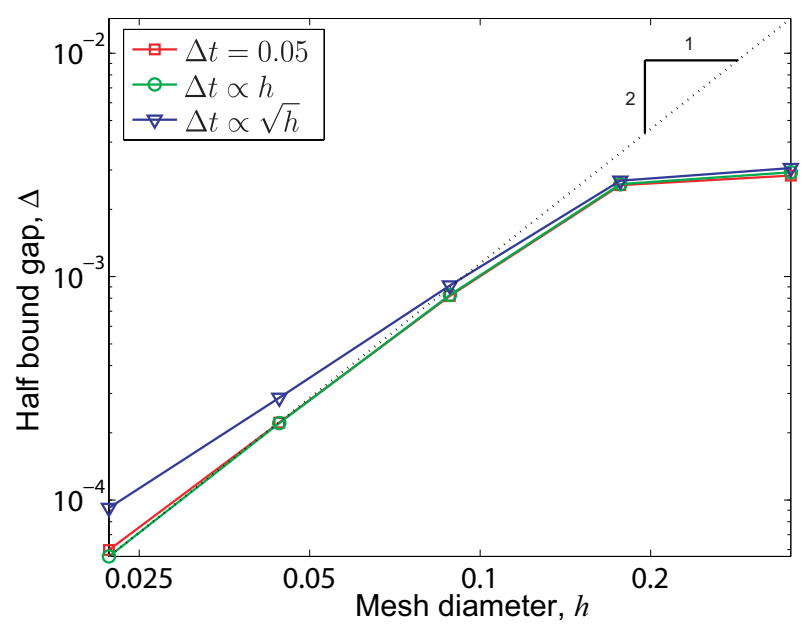

Figure 2: Example 1: Convergence of the half bound gap in a series of uniformly refined meshes of linear triangular elements $(p=1)$ using different strategies to determine the time step $\Delta t$.

in space for the quantity of interest is $\mathcal{O}\left(h^{2}\right)$ which is approximately obtained when the time step is taken to be $\Delta t=h$. If a constant time step is considered, the bounds have the expected rate of convergency for the initial meshes but in the last step the time discretization error is 
no longer negligible in front of the space discretization error. The strategy which considers $\Delta t \propto \sqrt{h}$ does not reach the expected rate of convergence and shows that in this case the convergence of the half bound gap is determined by the time step $\Delta t$ and not by the mesh size $h$. This shows that in order to asymptotically approach the optimal finite element convergence rate in space, the time step $\Delta t$ has to be chosen carefully.

The bounds obtained with the strategy $\Delta t \propto h$ are displayed in table 1 and in figure 3. Also the bound average $s^{\text {ave }}$ and the approximation of the output associated with the $\operatorname{cG}(p) \mathrm{dG}(q)$ approximation of $u, L^{\mathcal{O}}\left(\hat{u}_{h}\right)$, are shown. Note that, in this example, the bound average provides a better approximation of the output $L^{\mathcal{O}}(u)$ even for the coarser meshes.

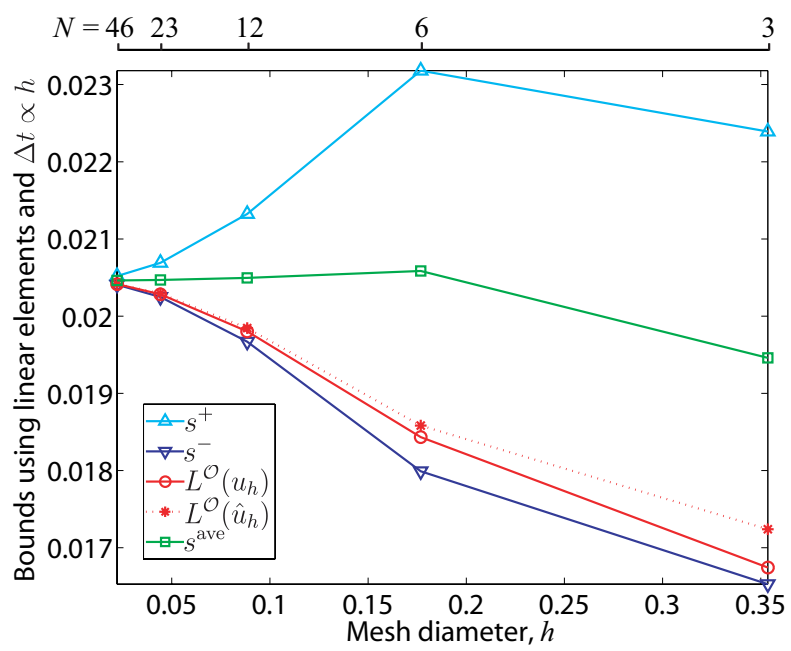

Figure 3: Example 1: Bounds obtained in a series of uniformly $h$-refined meshes using linear triangular elements using the time step $\Delta t=h$.

\begin{tabular}{cc|cc|ccccc}
\hline & & \multicolumn{7}{|c}{ linear elements } \\
\hline$n_{\mathrm{el}}$ & $N$ & $L^{\mathcal{O}}\left(\hat{u}_{h}\right)$ & $L^{\mathcal{O}}\left(u_{h}\right)$ & $s^{-}$ & $s^{+}$ & $s^{\text {ave }}$ & $\Delta$ & $\Delta_{\text {rel }}$ \\
\hline 8 & 3 & 0.017239 & 0.016743 & 0.016528 & 0.022392 & 0.019460 & 0.002932 & $15.07 \%$ \\
32 & 6 & 0.018582 & 0.018433 & 0.017991 & 0.023181 & 0.020586 & 0.002595 & $12.60 \%$ \\
128 & 12 & 0.019845 & 0.019803 & 0.019669 & 0.021323 & 0.020496 & 0.000827 & $4.03 \%$ \\
512 & 23 & 0.020295 & 0.020282 & 0.020248 & 0.020690 & 0.020469 & 0.000221 & $1.08 \%$ \\
2048 & 46 & 0.020419 & 0.020416 & 0.020407 & 0.020519 & 0.020463 & 0.000056 & $0.27 \%$ \\
\hline
\end{tabular}

Table 1: Example 1: results in a series of uniformly $h$-refined meshes using linear triangular elements and using the time step $\Delta t=h$.

The results for quadratic elements $(p=2)$ are shown in figure 4 for four cases: 1$)$ piecewise linear interpolation in time $(q=1)$ with a constant time step $\Delta t=0.002(N=50), 2)$ linear interpolation $q=1$ for a constant time step $\Delta t=0.00025(N=400)$, 3) linear interpolation $q=1$ and varying the time step $\Delta t \propto h^{2}$ and 4) quadratic interpolation $q=2$ for a varying time step $\Delta t \propto h$. For linear elements, $p=1$, if a constant time step is considered, the bounds have the expected rate of convergence for the initial meshes but deteriorate as the importance 
of the time discretization error increases with respect to the spatial discretization error (when the time discretization error is no longer negligible in front of the space discretization error). In particular, if the time step is kept constant the bound gap does not converge to zero with $h$. The strategy taking $\Delta t_{n} \propto h^{2}$ recovers the expected rate of convergencs, but it requires the use of a non reasonable amount of time steps (this could be precluded using a non-uniform time discretization). Finally, using a quadratic interpolation both in space and time $p=q=2$ and $\Delta t \propto h$ the desired convergency rate $\mathcal{O}\left(h^{4}\right)$ is not reached. This is due probably to two facts: first, a slight drop off in the rate of convergence may be due to the use of linear equilibrated fluxes for $\lambda_{n j}^{P}$ and $\lambda_{n j}^{D}$. Second, the convergence rate is possibly determined by the time step $\Delta t$ and not by the mesh size $h$. However, this strategy allows obtaining reasonably good results with few time steps.

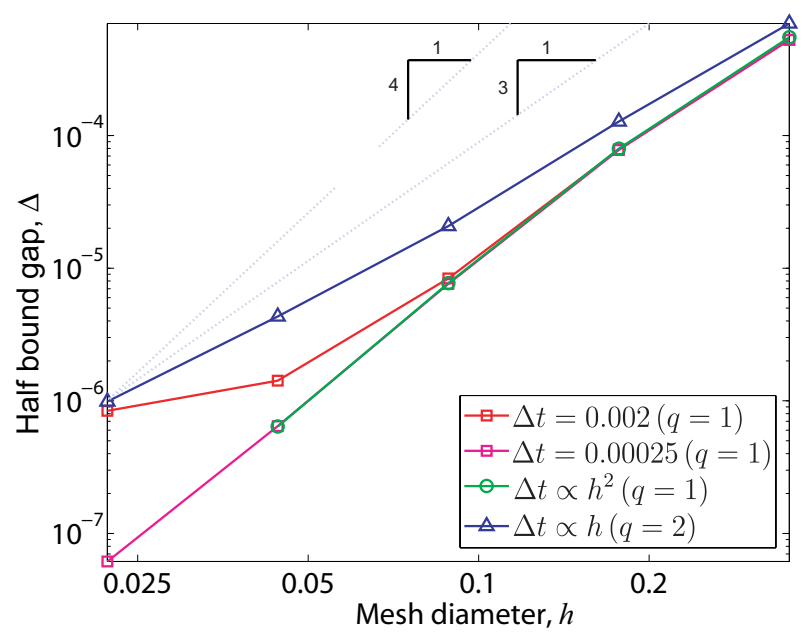

Figure 4: Example 1: Convergence of the half bound gap in a series of uniformly $h$-refined meshes using quadratic triangular elements $(p=2)$, using different strategies to determine the time step $\Delta t$.

\begin{tabular}{cc|cc|ccccc}
\hline & & \multicolumn{7}{|c}{ quadratic elements } \\
\hline$n_{\mathrm{el}}$ & $N$ & $L^{\mathcal{O}}\left(\hat{u}_{h}\right)$ & $L^{\mathcal{O}}\left(u_{h}\right)$ & $s^{-}$ & $s^{+}$ & $s^{\text {ave }}$ & $\Delta$ & $\Delta_{\text {rel }}$ \\
\hline 8 & 3 & 0.019892 & 0.019224 & 0.019505 & 0.020906 & 0.020205 & 0.000701 & $3.47 \%$ \\
32 & 6 & 0.020366 & 0.020185 & 0.020286 & 0.020541 & 0.020414 & 0.000128 & $0.62 \%$ \\
128 & 12 & 0.020452 & 0.020404 & 0.020433 & 0.020475 & 0.020454 & 0.000021 & $0.10 \%$ \\
512 & 23 & 0.020461 & 0.020447 & 0.020456 & 0.020464 & 0.020460 & 0.000004 & $0.02 \%$ \\
2048 & 46 & 0.020462 & 0.020458 & 0.020460 & 0.020462 & 0.020461 & 0.000001 & $0.005 \%$ \\
\hline
\end{tabular}

Table 2: Example 1: series of uniformly $h$-refined meshes using a quadratic interpolation both in space and time, $p=q=2$ and $\Delta t=h$.

The results in the case of using both a quadratic interpolation in space and time $p=q=2$ combined with the use of $\Delta t \propto h$ are displayed in table 2 and figure 5. In this case the bound average again provides a better approximation for the output $L^{\mathcal{O}}(u)$ than $L^{\mathcal{O}}\left(u_{h}\right)$ and $L^{\mathcal{O}}\left(\hat{u}_{h}\right)$. It is worth noting that in this case $s^{-} \geq L^{\mathcal{O}}\left(u_{h}\right)$ due to the term $R^{\mathrm{P}}\left(\psi_{h}\right)$ which in this case is a positive number appearing in the expression of the bounds. 


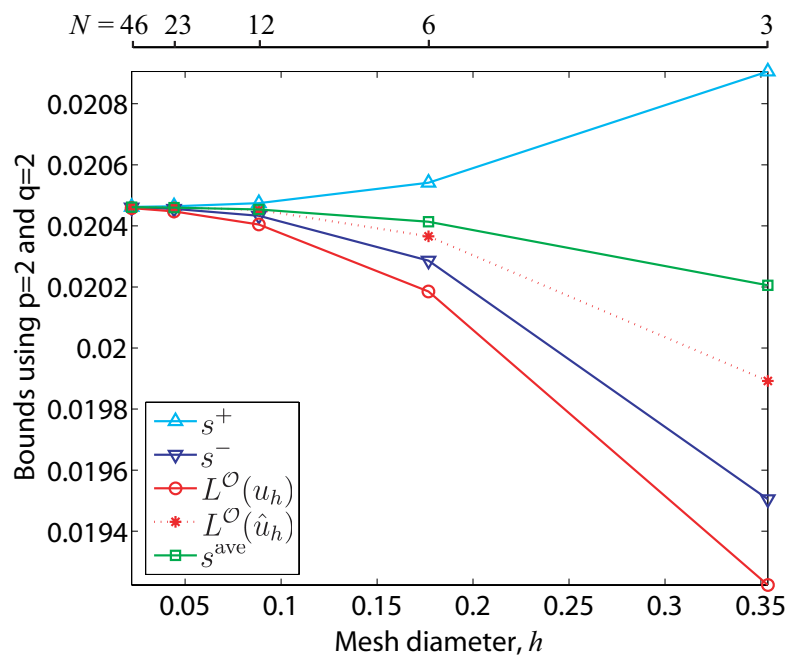

Figure 5: Example 1: Bounds in a series of uniformly $h$-refined meshes using a quadratic interpolation both in space and time, $p=q=2$ and $\Delta t=h$.

\subsection{Example 2: composite material}

The unsteady heat conduction problem is solved in the domain described in figure 6 for a nonuniform (composite) material. The problem is purely diffusive $(\sigma=0$ and $\boldsymbol{\alpha}=0)$. The thermal conductivity is smaller in the rectangular inclusions $(\nu=0.01)$ and larger for the bulk material $(\nu=1)$. The specimen is assumed to be thermally isolated on the lateral sides, that is homogeneous Neumann boundary conditions are prescribed in this part of the boundary. The temperature is set to zero on the top $(u=0)$ and a prescribed heat flux is imposed on the bottom, $\Gamma_{b}$, that is $\nabla u \cdot \boldsymbol{n}=g_{\mathrm{N}}(t)$, where

$$
g_{\mathrm{N}}(t)= \begin{cases}4 t(1-t)+1 & \text { for } t \in[0,1] \\ 4(1-t)(2-t)+1 & \text { for } t \in(1,2]\end{cases}
$$

The initial thermal state is assumed to be $u(0)=0$ and the time interval is taken from $t=0$ to $t=T=2$.

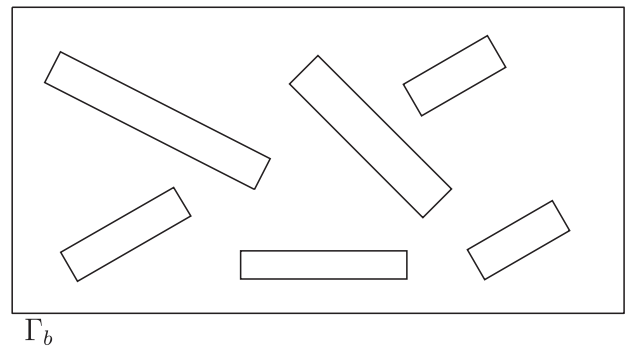

Figure 6: Example 2: Composite domain $\Omega$ : inside the rectangles, $\Omega_{2}$, the thermal conductivity is $\nu=0.01$ and in the remainder of the domain, $\Omega_{1}, \nu=1$.

Here, both space and time discretizations are quadratic, that is $p=2$ and $q=2$. The selected output of interest is the average of the temperature on the bottom, $\Gamma_{b}$ :

$$
L^{\mathcal{O}}(u)=\frac{1}{\operatorname{meas} \Gamma_{b}} \int_{0}^{T} \int_{\Gamma_{b}} u(x, y, t) \mathrm{d} \Gamma \mathrm{d} t .
$$


This quantity of interest is represented by a Neumann boundary condition for the dual problem $g_{\mathrm{N}}^{\mathcal{O}}(t)=1 / \operatorname{meas}\left(\Gamma_{b}\right)$ on $\Gamma_{b}$.

An adaptive procedure is carried out subdividing the elements with larger values of $\Delta_{k}$ (larger contributions to the bound gap). The bounds are obtained using a fourth order interpolation for the dual estimates, $r=4$. The results along the adaptive process are shown in figure 7 , where the representative mesh size $h$ is defined as $h=\sqrt{1 / n_{\mathrm{el}}}$. At each remeshing step
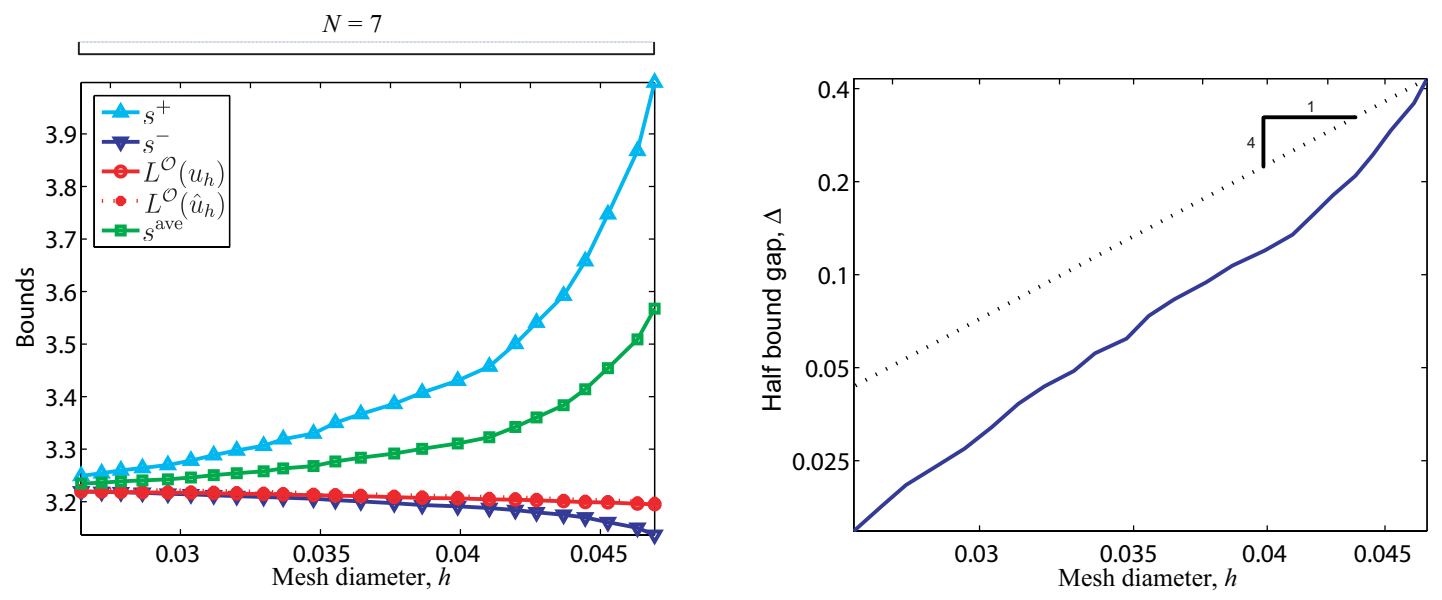

Figure 7: Example 2: Computed bounds for an adaptive $h$-refinement using a quadratic interpolation both in space and time $p=q=2$ (left) and convergence of the half bound gap (right).

$1 \%$ of the elements are marked to be refined. For each step a constant time step is considered $\Delta t_{n}=\Delta t$ and the time step is reduced along the adaptive loop such that the convergence rate is driven by the space discretization error. That is, in the adaptive steps where the rate of convergence of the half bound gap with respect to the space discretization is not the expected one (due to a non-negligible time discretization error), the time step $\Delta t$ is divided by two. The adaptive procedure is then continued until the relative bound gap reaches a target value of $0.5 \%$, that is, $\Delta_{\text {rel }} \leq 0.005$. The adaptive procedure starts with $N=7$ and the time step does not need to be modified in all the steps. Note that in this example since the time discretization error is very small, $L^{\mathcal{O}}\left(u_{h}\right) \approx L^{\mathcal{O}}\left(\hat{u}_{h}\right)$. Moreover, the lower bound estimates provide better approximation than the upper bound and the bound average.

Table 3 summarizes the results for the first and final iteration and the initial and final meshes are shown in figure 8 . The adaptive procedure guarantees a relative half bound gap less than $0.5 \%$ using only $N=7$. For the final mesh an extra computation has been done with a lower time step, using $n=10$ and $N=20$ to compare the resulting bounds. The error in the final mesh with 1429 elements is mainly due to the space discretization error: decreasing the time step does not practically vary the output and yields only a slight improvement of the bounds.

\subsection{Example 3: quasi-2D transport}

This example is the transient version of a steady quasi-2D transport problem introduced in [18]. The effect of including the convective term is analyzed in this simple problem for different values of the velocity $\boldsymbol{\alpha}$. Equation (1) is solved in the unit square, $\Omega=[0,1] \times[0,1]$, for $\nu=1$, $\sigma=1$ and a uniform horizontal velocity field $\boldsymbol{\alpha}=(\alpha, 0)$. The performance of the introduced 


\begin{tabular}{cc|cc|cccc}
\hline$n_{\mathrm{el}}$ & $N$ & $L^{\mathcal{O}}\left(\hat{u}_{h}\right)$ & $L^{\mathcal{O}}\left(u_{h}\right)$ & $s^{-}$ & $s^{+}$ & $s^{\text {ave }}$ & $\Delta_{\text {rel }}$ \\
\hline 454 & 7 & 3.19971 & 3.19517 & 3.13694 & 3.99799 & 3.56747 & $12.07 \%$ \\
1429 & 7 & 3.22388 & 3.21936 & 3.21962 & 3.24922 & 3.23442 & $0.46 \%$ \\
1429 & 10 & 3.22404 & 3.22144 & 3.22068 & 3.24844 & 3.23456 & $0.43 \%$ \\
1429 & 20 & 3.22404 & 3.22310 & 3.22135 & 3.24775 & 3.23455 & $0.41 \%$ \\
\hline
\end{tabular}

Table 3: Example 2: results in a series of adaptive $h$-refinement.
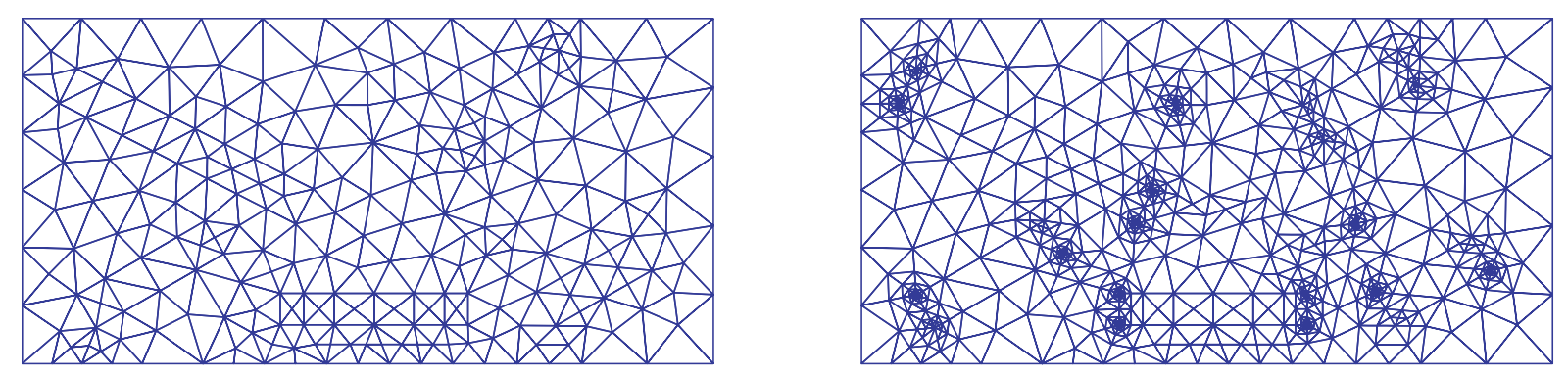

Figure 8: Example 2: Initial (left) and final meshes (right) of the adaptive procedure with $n_{\mathrm{el}}=454$ and 1429 respectively.

estimates is tested for different values of $\alpha$. The boundary conditions are of Dirichlet type on the lateral sides, homogeneous on the right $u(1, y)=0$ and set to 1 on the left $u(0, y)=1$. The boundary condition on both the top and bottom are Neumann homogeneous. The source term is $f=0$, and the initial condition is $u_{0}(x, y)=1-x$. Time integration is performed to $t=T=1$. The degrees of the space and time interpolations are $p=1, q=1$ and $r=4$.

The quantity of interest is an overall average of the solution, that is

$$
L^{\mathcal{O}}(u)=\int_{0}^{T} \int_{\Omega} u(x, y, t) \mathrm{d} \Omega \mathrm{d} t,
$$

which corresponds to $f^{\mathcal{O}}=1$.

The error estimation strategies and the computation of bounds are performed for a series of uniformly $h$-refined meshes with $\Delta t=h$ and different values of $\alpha$. The results are displayed in table 4 and figure 9. For all the values of $\alpha$, the rate of convergence of the bound gap is found to be equal to the expected one for the error, that is $\mathcal{O}\left(h^{2}\right)$. It is worth noting that the bound gap is larger as $\alpha$ increases. For $\alpha=100$ the bound gap is 4 orders of magnitude larger than the for $\alpha=0$, being the quantity of interest of the same order. The convergence rate in all the cases is clearly determined by the spatial error. Moreover, although the bounds account both for the discretization error in space and time, the results are nearly as sharp as the bounds obtained in [15] which only considers the contribution of the spatial error.

This increment in the bound gap does not correspond to the actual error increment and therefore it has to be concluded that the efficiency of the computed error bounds is deteriorated if the convection parameter is large.

In order to check the convergence in time of the proposed algorithm a $\Delta t$-refining procedure is carried out. A fixed spatial mesh composed by 2048 triangular elements (half squares) and an initial constant time step $\Delta t=0.5$ are considered to start the process. In each refinement step, the time step $\Delta t$ is divided by two. The results are displayed in figure 10 . The convergence of 


\begin{tabular}{cc|cc|cc|cc|cc}
\hline & & \multicolumn{2}{|c|}{$\boldsymbol{\alpha}=0$} & \multicolumn{2}{c|}{$\boldsymbol{\alpha}=1$} & \multicolumn{2}{c|}{$\boldsymbol{\alpha}=5$} & \multicolumn{2}{c}{$\boldsymbol{\alpha}=10$} \\
\hline$n_{\mathrm{el}}$ & $N$ & $s^{\text {ave }}$ & $\Delta$ & $s^{\text {ave }}$ & $\Delta$ & $s^{\text {ave }}$ & $\Delta$ & $s^{\text {ave }}$ & $\Delta$ \\
\hline 32 & 6 & 0.466028 & 0.001717 & 0.532588 & 0.002032 & 0.738888 & 0.036791 & 0.848585 & 0.171335 \\
128 & 12 & 0.465682 & 0.000414 & 0.532864 & 0.000488 & 0.739246 & 0.008675 & 0.849791 & 0.040686 \\
512 & 23 & 0.465587 & 0.000103 & 0.532940 & 0.000120 & 0.739414 & 0.002056 & 0.849588 & 0.009456 \\
2048 & 46 & 0.465562 & 0.000026 & 0.532959 & 0.000030 & 0.739465 & 0.000493 & 0.849582 & 0.002212 \\
8192 & 91 & 0.465555 & 0.000006 & 0.532964 & 0.000007 & 0.739479 & 0.000121 & 0.849585 & 0.000530 \\
\hline
\end{tabular}

Table 4: Example 3: results in a series of uniformly $h$-refined meshes with $\Delta t=h$.

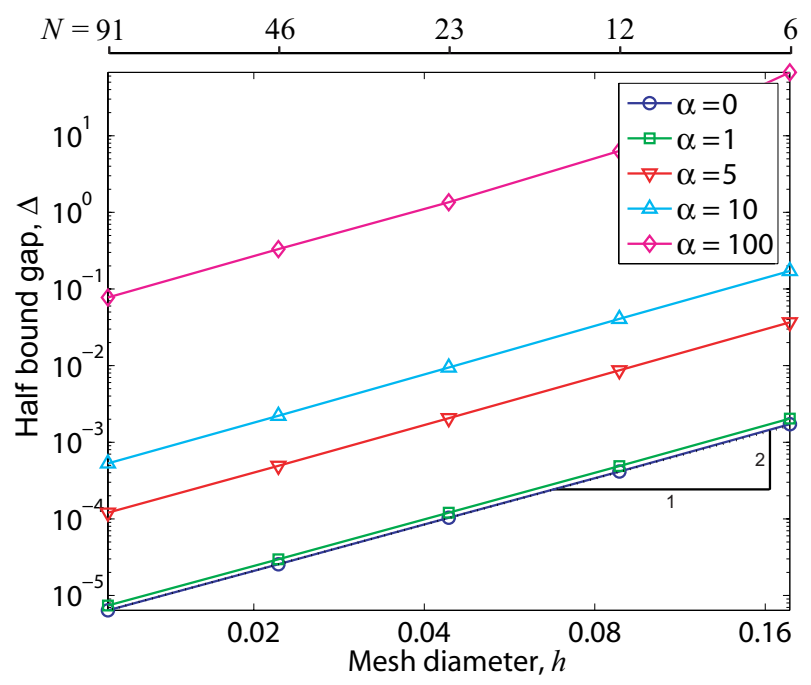

Figure 9: Example 3: convergence of the half bound gap for different convection parameters $\boldsymbol{\alpha}=0,1,5,10$ and 100 .

the bound gap degenerates as the value of $\alpha$ increases at the same time that the bound gap is larger for larger values of $\alpha$. For $\alpha=100$ the bound gap is 4 orders of magnitude larger than the for $\alpha=0$, being the quantity of interest of the same order.

The bounds obtained with the algorithm presented in the first part I of this work [15] are compared with the results of the present algorithm. Figure 10 shows that the bounds which only account for the spatial error (non-strict bounds) are nearly insensitive to the change of the time step. In fact, if the convergence of the bounds which do not account for the time discretization error is plotted for each value of $\alpha$, it can be seen that the bounds increase as the time step is reduced (although the rise of the half bound gap is barely perceptible as compared to the variations of the bounds accounting for both the error in space and time).

Figure 11 shows bounds obtained for the values of the convection parameter $\alpha=0,5$ and 100. For very large time steps the smoothed approximation $L^{\mathcal{O}}\left(u_{h}\right)$ is not a good approximation of $L^{\mathcal{O}}(u)$ (in comparision with the $\operatorname{cG}(p) \mathrm{dG}(q)$ approximation $L^{\mathcal{O}}\left(\hat{u}_{h}\right)$ ). However, for $\alpha=0$ and 5 , the procedure yields quite competitive bounds taking into account that the starting point of the procedure is $L^{\mathcal{O}}\left(u_{h}\right)$. Therefore, in this case for low values of $\alpha$ the quality of the bounds is determined by the quality of the smoothed approximations. For large values of $\alpha$, although the smoothed approximations are worst that the $\mathrm{cG}(p) \mathrm{dG}(q)$ approximation, the bounds are 


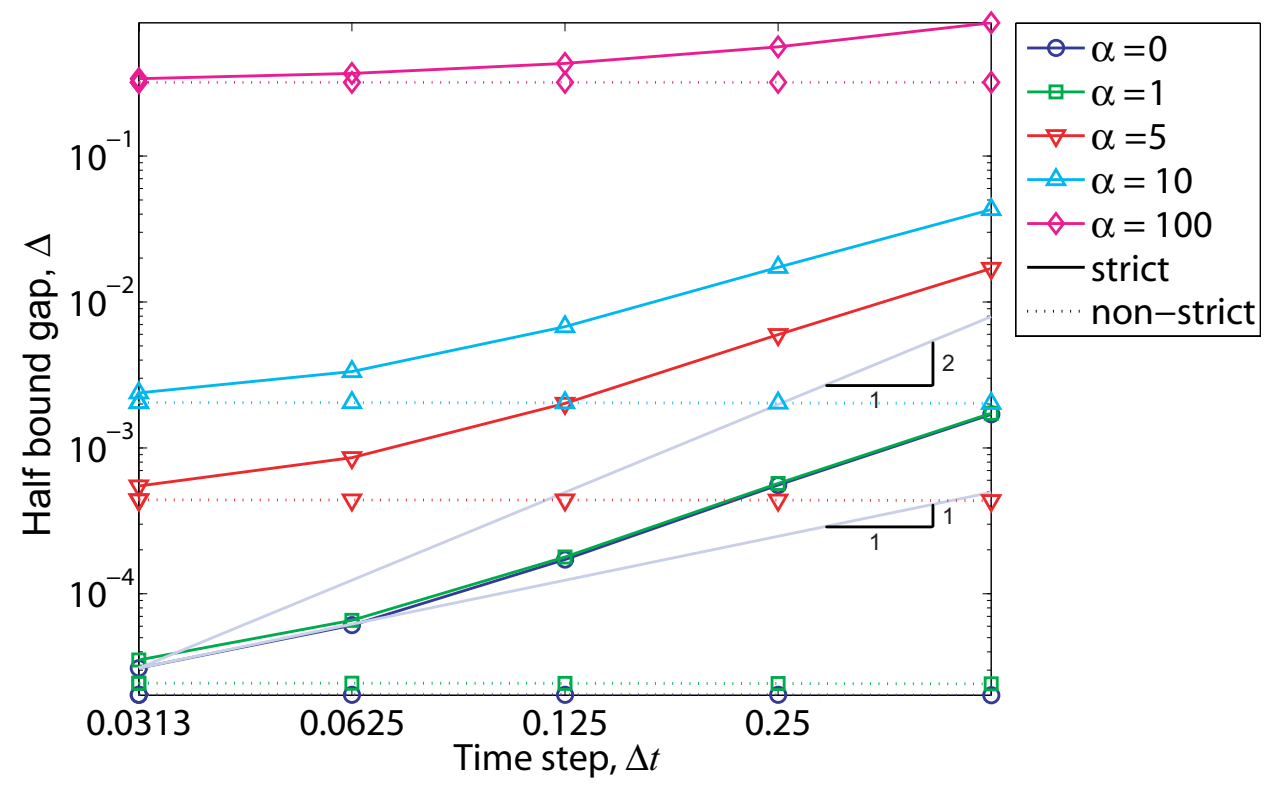

Figure 10: Example 3: convergence with respect to the time discretization of the half bound gap for different convection parameters $\boldsymbol{\alpha}=0,1,5,10$ and 100 using both the methodology presented in this paper and in the first part [15].

clearly deteriorated due to the convection-dominated nature of the problem.
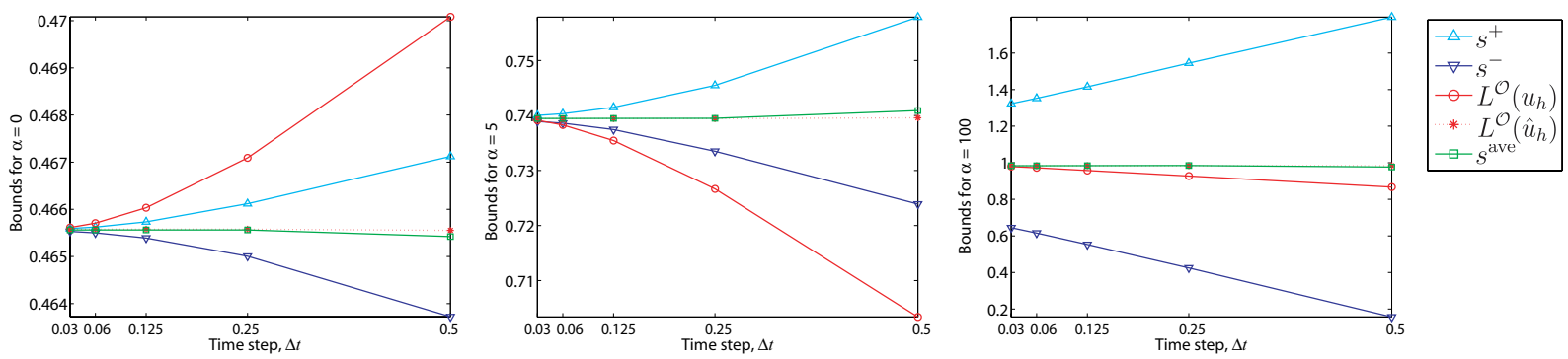

Figure 11: Example 3: bounds accouting both for the error in space and time for different convection parameters $\boldsymbol{\alpha}=0,5$ and 100 with respect to a uniform refinement in time.

\subsection{Example 4: rotating transport}

Again, a transient version of a steady problem analyzed in [18] is considered. The computational domain is $\Omega=[0,1] \times[0,1]$ and the model parameters are $\nu=1, \sigma=10$ and $\boldsymbol{\alpha}=250\left(y-\frac{1}{2}, \frac{1}{2}-x\right)$. The boundary conditions are Dirichlet homogeneous on the whole boundary $\partial \Omega$ and the initial condition is $u_{0}=0$. A localized source term is $f=100$ in the square $[0.7,0.8] \times[0.7,0.8]$ and $f=0$ elsewhere, see figure 12 . The output of interest is a local average in the square region $[0.2,0.3] \times[0.2,0.3]$, that is $f^{\mathcal{O}}=1$ in $[0.2,0.3] \times[0.2,0.3]$ and $f^{\mathcal{O}}=0$ elsewhere. The parameters describing the space-time discretization are $p=1$ and $q=1$, and for a final time $T=0.03$. 


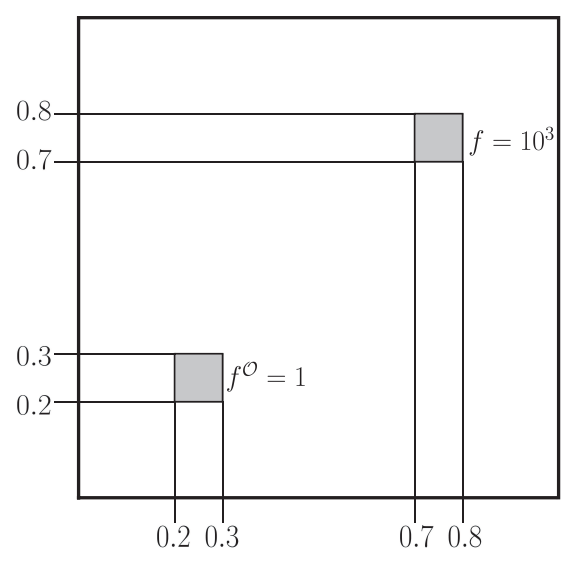

Figure 12: Example 4: Rotating transport forcing and output regions.

A series of adapted meshes is produced by subdividing at each remeshing step $4 \%$ of the elements, those with the larger contributions to the bound gap. Also the time step $\Delta t$ is adjusted (is divided by two in the iterations where a slow convergence of the half bound gap is observed). The adaptive procedure starts with a mesh of 322 elements and with $N=6$ and stops when the half bound gap reaches the target value of 0.000006 , that is, $\Delta \leq 6 \cdot 10^{-6}$, see figure 13 .
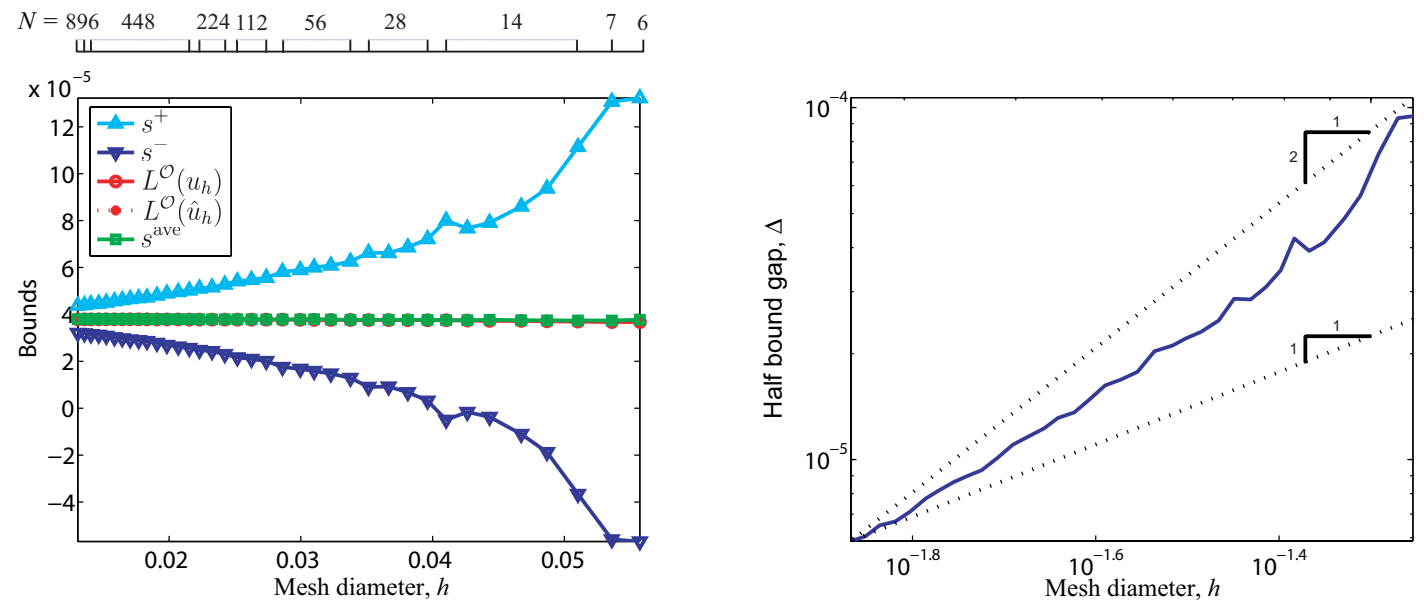

Figure 13: Example 4: Error bounds for an adaptive $h$-refinement and adjusting the constant time step $\Delta t$.

The initial mesh of 322 elements and $N=6$ certifies a wide interval for the quantity of interest, $L^{\mathcal{O}}\left(u_{\tau}\right)=3.77287 \cdot 10^{-5} \pm 9.45362 \cdot 10^{-5}$, after remeshing the bounds associated with the final mesh of 5855 elements and $N=896$ set a much narrower interval, $L^{\mathcal{O}}\left(u_{\tau}\right)=$ $3.79554 \cdot 10^{-5} \pm 0.58690 \cdot 10^{-5}$, see table 5 . The primal and adjoint solutions at the final computational times ( $t=T$ for the primal and $t=0$ for the adjoint) are displayed in figure 14 . The local elementary contributions $\Delta_{k}$ to the global bound gap are plotted in figure 15 for the initial mesh and for an intermediate mesh of the adaptive procedure. The larger values of the local contributions are precisely in the zones where either the primal or the adjoint solutions have larger gradients. Also in figure 15 the resulting final mesh is displayed. 


\begin{tabular}{cc|c|rrrc}
\hline$n_{\mathrm{el}}$ & $N$ & $L^{\mathcal{O}}\left(u_{h}\right)$ & \multicolumn{1}{c}{$s^{-}$} & \multicolumn{1}{c}{$s^{+}$} & \multicolumn{1}{c}{$s^{\text {ave }}$} & $\Delta$ \\
\hline 322 & 6 & $3.677 \cdot 10^{-5}$ & $-5.681 \cdot 10^{-5}$ & $13.226 \cdot 10^{-5}$ & $3.773 \cdot 10^{-5}$ & $9.454 \cdot 10^{-5}$ \\
5855 & 896 & $3.792 \cdot 10^{-5}$ & $3.209 \cdot 10^{-5}$ & $4.382 \cdot 10^{-5}$ & $3.796 \cdot 10^{-5}$ & $0.587 \cdot 10^{-5}$ \\
\hline
\end{tabular}

Table 5: Example 4: results for the initial and final meshes of the adaptive $h$-refinement procedure.
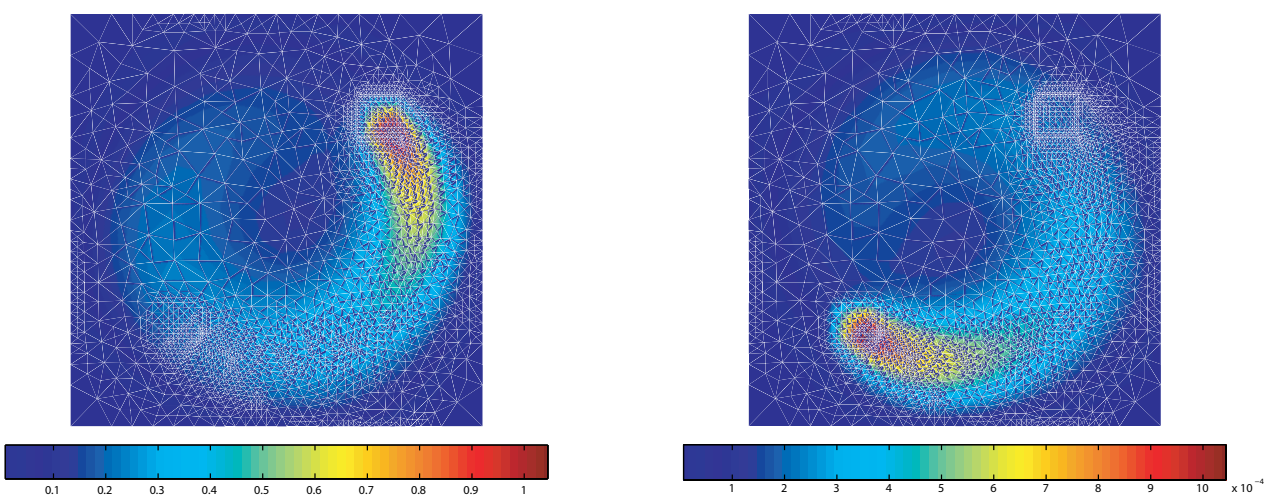

Figure 14: Example 4: Primal and adjoint solution at the final time: $t=T$ for the primal and $t=0$ for the adjoint.
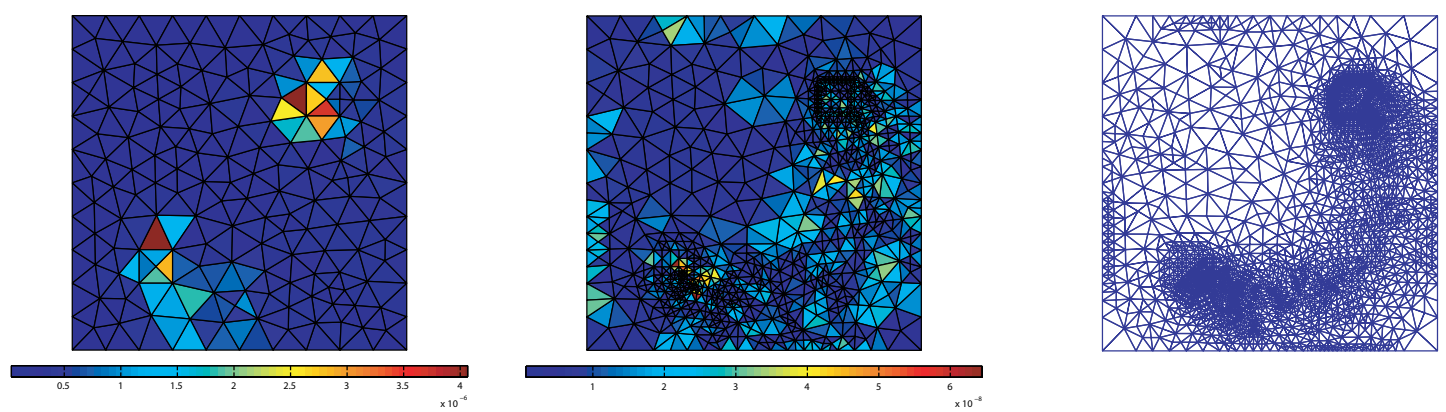

Figure 15: Example 4: Elementary contributions to the bound gap in initial mesh (left) and intermediate mesh with $n_{\mathrm{el}}=1577$ (center). Final adapted mesh (right).

\subsection{Example 5: canister}

The final example represents the transport of pollutant inside an active carbon filter. The transient convection-reaction-diffusion equation is solved in the simplified canister geometry shown in figure 16. The diffusion is constant $\nu=0.01$ whereas the reaction is larger in the outlet of the canister $\left(\sigma=10\right.$ in $\left.\Omega_{2} \cup \Omega^{\mathcal{O}}\right)$ and smaller in the rest of the canister $(\sigma=0.1$ in $\Omega_{1}$ ). Thus, $\Omega^{\mathcal{O}}$ is a pollutant trap capturing all the pollutant that the actual filter (domain $\Omega_{1}$ ) is not able to retain. The advection field, $\boldsymbol{\alpha}$, is a piecewise linear field (see figure 16) resulting from a finite element computation of a potential flow in the same mesh. The inlet concentration of pollutant is set to one ( $u=1$ in $\left.\Gamma_{1}\right)$ and the outlet concentration of pollutant is set to zero $\left(u=0\right.$ in $\left.\Gamma_{2}\right)$. The rest of the boundary conditions are Neumann homogeneous because the walls of the canister are considered to be impermeable.

The initial pollutant concentration state is taken as $u_{0}=1$ in the inlet boundary, $\Gamma_{1}$, and zero elsewhere. In practice, the initial condition $u_{0}$ has to be interpolated in the mesh and 

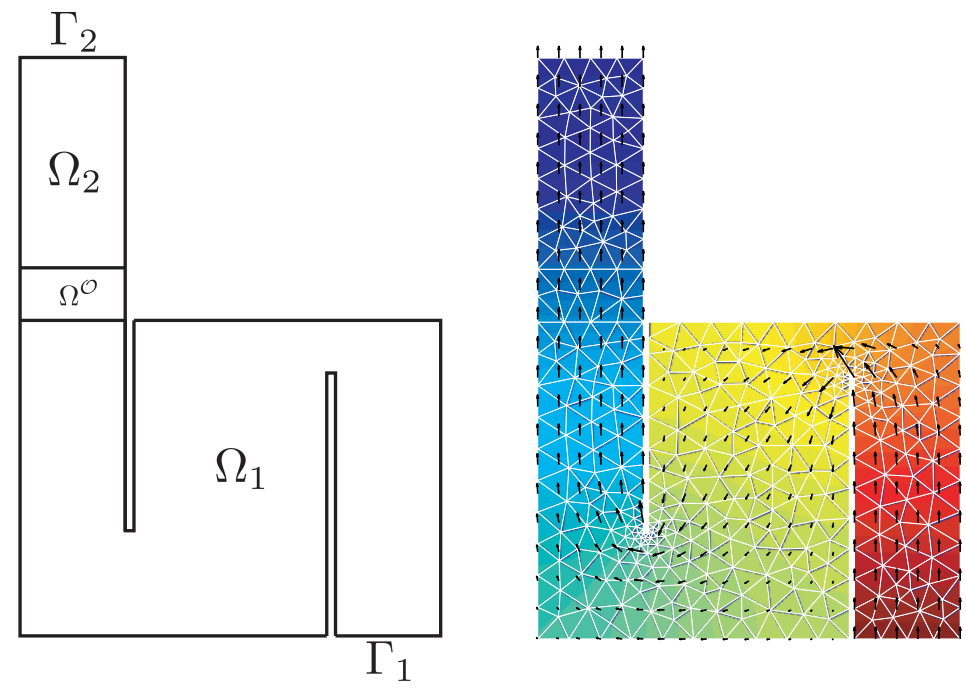

Figure 16: Example 5: Computational domain (left) and incompressible advection field $\boldsymbol{\alpha}$ (right).

therefore $u_{0}$ is set to 1 in the nodes of the mesh lying in $\Gamma_{1}$ and set to zero in the rest of the nodes. The time interval is taken from $t=0$ to $t=T=2$.

The quantity of interest is the total pollutant captured by the trap domain $\Omega^{\mathcal{O}}$ along the complete time evolution. Note that the canister is considered to work properly if this quantity is small enough. If the outcome of pollutant exceeds a threshold value, the canister breaks and the design is not admissible. This quantity is expressed in terms of the solution by

$$
L^{\mathcal{O}}(u)=\int_{0}^{T} \int_{\Omega^{\mathcal{O}}} u(x, y, t) \mathrm{d} \Omega \mathrm{d} t,
$$

that is $u_{T}^{\mathcal{O}}=0$ and $f^{\mathcal{O}}=1$ in $\Omega^{\mathcal{O}}$ and zero elsewhere, in equation (5).

Two different strategies for space-time adaptation have been used in order to yield the desired accuracy. The first procedure yields the desired accuracy in two steps: first the time discretization error is neglected and the strategy proposed in [15] is used to obtain an optimal adapted mesh with respect to space. It is worth noting that the strategy proposed in [15] is cheaper since the approximations used as input of the error estimation procedure verify the Galerkin orthogonality property. For this final mesh, the strategy proposed in this work along with a uniform refinement of the constant time step $\Delta t$ is used to guarantee the bounds for $L^{\mathcal{O}}(u)$, taking into account both the space and time discretization errors. The second strategy is to use the space-time adaptation also used in the previous sections (in all the intermediate steps the bounds are strict for $L^{\mathcal{O}}(u)$ and the time step is adjusted in each step of the adaptive procedure).

The starting mesh of the uniform $\Delta t$-refinement procedure (obtained using the strategy given in [15] for a target relative half bound gap of 2.5\%) contains 5786 elements, see figure 17. Here, both the spatial and time discretizations are linear, that is $p=1$ and $q=1$, and the bounds are obtained using a fourth order interpolation for the dual estimates $r=4$. The results are displayed in figure 17 and in table 6. As expected, for large values of $N$ the bounds present a drop off in the convergence as the contribution of the spatial discretization error acquires importance with respect to the error in time. 

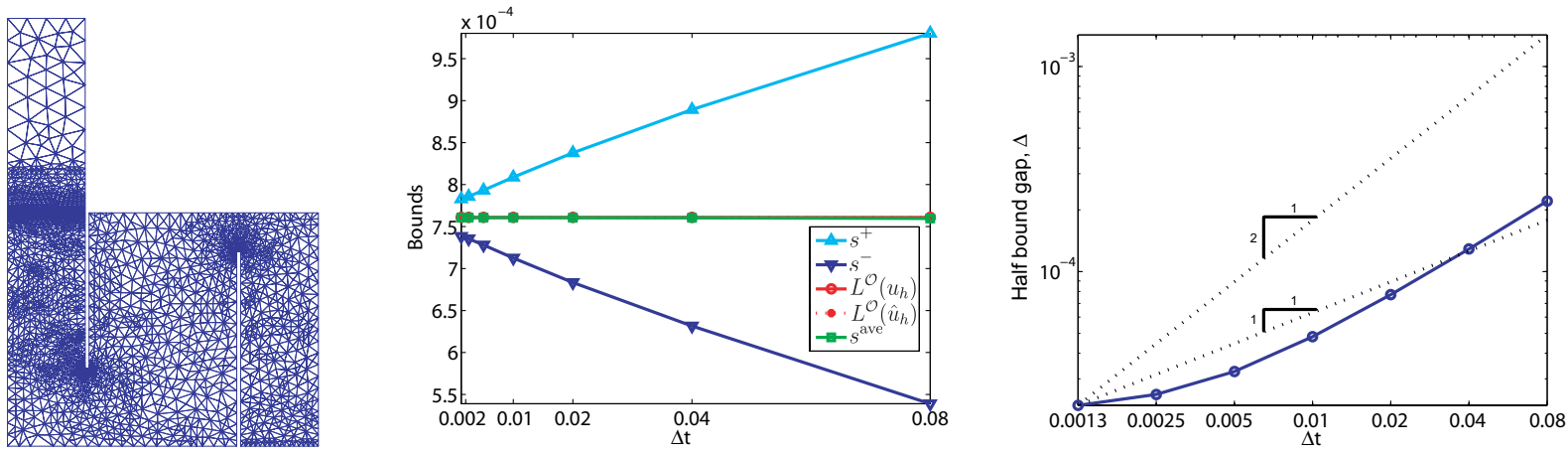

Figure 17: Example 5: starting mesh for the uniform $\Delta t$-refinement obtained with the strategy given in [15] for a target relative half bound gap of $2.5 \%$ (left), computed bounds for the $\Delta t$ refinement (center) and its convergence (right).

\begin{tabular}{c|c|ccccc}
\hline$N$ & $L^{\mathcal{O}}\left(u_{h}\right)$ & $s^{-}$ & $s^{+}$ & $s^{\text {ave }}$ & $\Delta$ & $\Delta_{\text {rel }}$ \\
\hline 25 & $7.609 \cdot 10^{-4}$ & $5.390 \cdot 10^{-4}$ & $9.802 \cdot 10^{-4}$ & $7.596 \cdot 10^{-4}$ & $2.206 \cdot 10^{-4}$ & $29.04 \%$ \\
50 & $7.609 \cdot 10^{-4}$ & $6.315 \cdot 10^{-4}$ & $8.893 \cdot 10^{-4}$ & $7.604 \cdot 10^{-4}$ & $1.289 \cdot 10^{-4}$ & $16.95 \%$ \\
100 & $7.609 \cdot 10^{-4}$ & $6.834 \cdot 10^{-4}$ & $8.378 \cdot 10^{-4}$ & $7.606 \cdot 10^{-4}$ & $0.772 \cdot 10^{-4}$ & $10.15 \%$ \\
200 & $7.609 \cdot 10^{-4}$ & $7.125 \cdot 10^{-4}$ & $8.088 \cdot 10^{-4}$ & $7.606 \cdot 10^{-4}$ & $0.482 \cdot 10^{-4}$ & $6.33 \%$ \\
400 & $7.609 \cdot 10^{-4}$ & $7.281 \cdot 10^{-4}$ & $7.932 \cdot 10^{-4}$ & $7.607 \cdot 10^{-4}$ & $0.326 \cdot 10^{-4}$ & $4.28 \%$ \\
800 & $7.609 \cdot 10^{-4}$ & $7.355 \cdot 10^{-4}$ & $7.858 \cdot 10^{-4}$ & $7.607 \cdot 10^{-4}$ & $0.252 \cdot 10^{-4}$ & $3.31 \%$ \\
1600 & $7.609 \cdot 10^{-4}$ & $7.384 \cdot 10^{-4}$ & $7.829 \cdot 10^{-4}$ & $7.607 \cdot 10^{-4}$ & $0.223 \cdot 10^{-4}$ & $2.93 \%$ \\
\hline
\end{tabular}

Table 6: Example 5: computed bounds for a uniform $\Delta t$-refinement for a constant spatial mesh of 5786 elements.

Also the convergence of the bounds is analayzed using the strategy presented in the previous examples. The adaptive procedure subdivides the elements with larger contribution to the bound gap and adjusts the time step in each iteration, until the relative half bound gap reaches a target value of $7 \%$. Also in this example, in each step, $1 \%$ of the elements are marked to be refined. In this case a linear interpolation in space $p=1$ and a quadratic interpolation in time $q=2$ are used, whereas the bounds are obtained using a fourth order interpolation for the dual estimates $r=4$. The initial and final meshes are shown in figure 18 .

The corresponding bounds are displayed in figure 19. In the first iteration $\left(n_{\mathrm{el}}=686\right.$ and $N=8$ ) the bounds guarantee that $L^{\mathcal{O}}(u)=7.678 \cdot 10^{-4} \pm 3.230 \cdot 10^{-4}=7.678 \cdot 10^{-4} \pm 42.07 \%$ whereas for the final mesh $\left(n_{\mathrm{el}}=2703\right.$ and $\left.N=825\right)$, the bounds for the quantity of interest guarantee that $L^{\mathcal{O}}(u)=7.606 \cdot 10^{-4} \pm 0.524 \cdot 10^{-4}=7.606 \cdot 10^{-4} \pm 6.89 \%$.

\section{Concluding remarks}

The methodologies presented in this series of two papers provide computable bounds for linear outputs of parabolic problems. In the first part the error associated with the time discretization is neglected and therefore the space adaptivity is sufficient to control the accuracy of the solution. The quality of the bounds is however degraded for large values of the advection parameter 

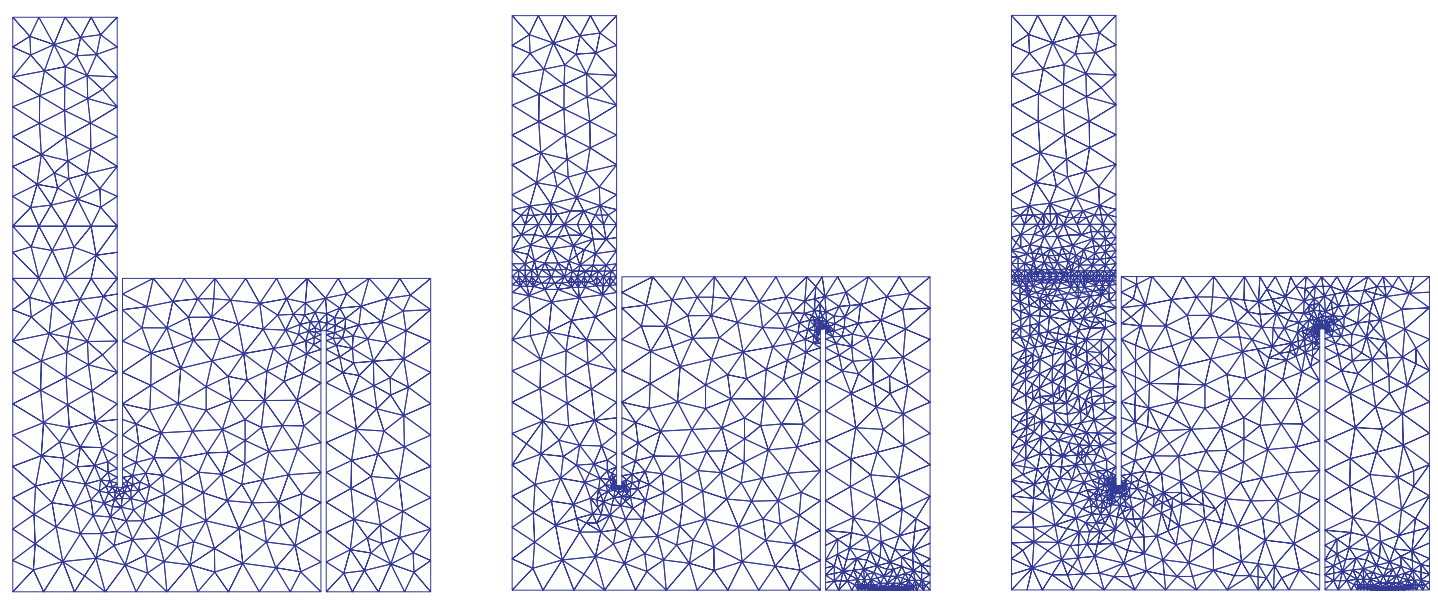

Figure 18: Example 5: initial, intermadiate and final meshes with 686, 1489 and 2703 elements respectively. The associated time steps are $N=8,109$ and 825 respectively.
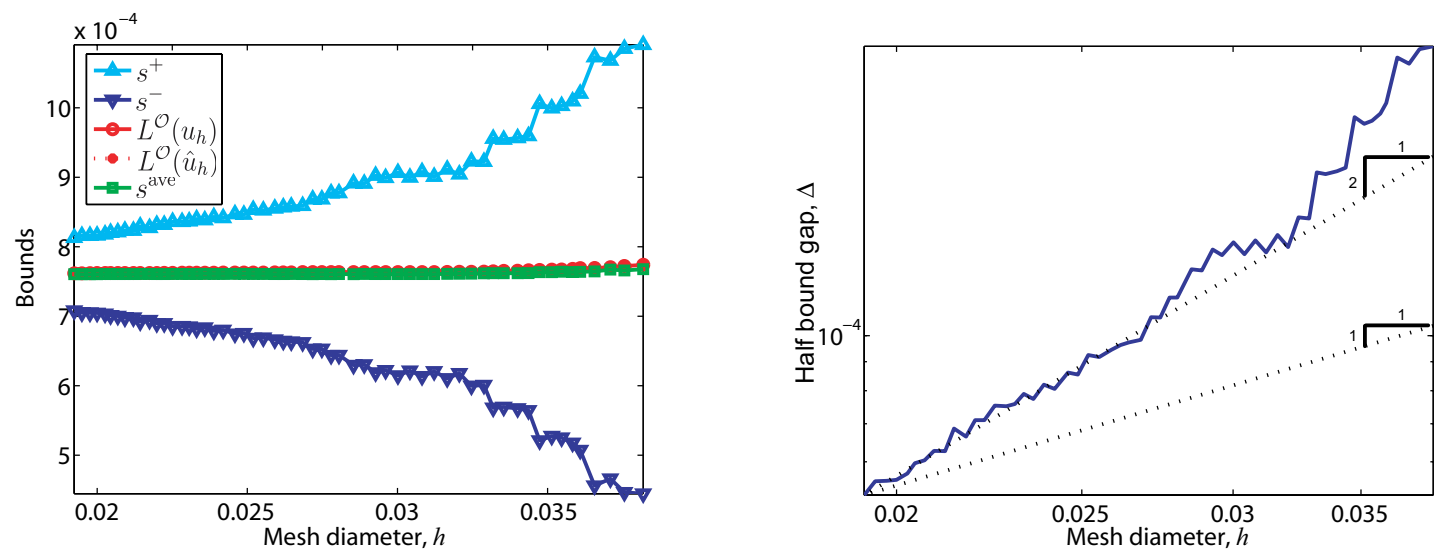

Figure 19: Example 5: Computed bounds for the adaptive space-time procedure (left) and convergence of the half bound gap (right).

because the error equations to be solved are symmetrized. In the second part, the methodology includes the assessment of the time error and, consequently, time adaptivity is also required to control the quality of the solution. In this second approach, the approximate solution has to be post-processed to enforce continuity. This smoothing is required to use the approximate solution as an input of the error assessment. The predicted convergence rates (of the nonsmoothed solution) are affected by this post-processing. The behavior of the obtained bounds is also degenerated for advection dominated problems.

The results demonstrate that the procedures introduced here are valuable tools to assess the quality of linear outputs in the context of parabolic problems. Nevertheless, many questions still require an answer and there is space for further research in this very same topic. For instance, considering non-symmetric error equations would possibly allow to better account for the advection effect. Moreover, the design of optimal adaptive procedures in this context is also a critical issue, both for the space and time discretizations. 


\section{A Proof of remark 2}

For any $v \in \mathcal{W}$, using the definitions of the bilinear form $A(\cdot, \cdot)$ and of the linear functional $L(\cdot)$, the primal residual can be rewritten as:

$$
\begin{aligned}
R^{\mathrm{P}}(v) & =L(v)-A\left(u_{h}, v\right) \\
& =\int_{0}^{T} \ell(t ; v) \mathrm{d} t+\left(u_{0}, v(0)\right)-\int_{0}^{T}\left[\left\langle\dot{u}_{h}, v\right\rangle+a\left(t ; u_{h}, v\right)\right] \mathrm{d} t-\left(u_{h}(0), v(0)\right) \\
& =\int_{0}^{T}\left[\left\langle f-\dot{u}_{h}, v\right\rangle-a\left(t ; u_{h}, v\right)\right] \mathrm{d} t,
\end{aligned}
$$

since the approximation $u_{h}$ verifies the initial condition $u_{h}(0)=u_{0}$.

Similarly, for any $v \in \mathcal{W}$, using the definitions of the bilinear form $A(\cdot, \cdot)$, of the linear functional $L^{\mathcal{O}}(\cdot)$, and with the help of the following equality

$$
\int_{0}^{T}\left\langle\dot{v}, \psi_{h}\right\rangle \mathrm{d} t=-\int_{0}^{T}\left\langle\dot{\psi}_{h}, v\right\rangle \mathrm{d} t+\left(v(T), \psi_{h}(T)\right)-\left(v(0), \psi_{h}(0)\right),
$$

the adjoint residual can be rewritten as:

$$
\begin{aligned}
R^{\mathrm{D}}(v)= & L^{\mathcal{O}}(v)-A\left(v, \psi_{h}\right) \\
= & \int_{0}^{T} \ell^{\mathcal{O}}(t ; v) \mathrm{d} t+\left(u_{T}^{\mathcal{O}}, v(T)\right)-\int_{0}^{T}\left[\left\langle\dot{v}, \psi_{h}\right\rangle+a\left(t ; v, \psi_{h}\right)\right] \mathrm{d} t-\left(v(0), \psi_{h}(0)\right) \\
= & \int_{0}^{T}\left[\left\langle f^{\mathcal{O}}, v\right\rangle-a\left(t ; v, \psi_{h}\right)\right] \mathrm{d} t+\left(u_{T}^{\mathcal{O}}, v(T)\right)-\left(v(0), \psi_{h}(0)\right) \\
& -\left[-\int_{0}^{T}\left\langle\dot{\psi}_{h}, v\right\rangle \mathrm{d} t+\left(v(T), \psi_{h}(T)\right)-\left(v(0), \psi_{h}(0)\right)\right] \\
= & \int_{0}^{T}\left[\left\langle f^{\mathcal{O}}, v\right\rangle+\left\langle\dot{\psi}_{h}, v\right\rangle-a\left(t ; v, \psi_{h}\right)\right] \mathrm{d} t+\left(u_{T}^{\mathcal{O}}, v(T)\right)-\left(v(T), \psi_{h}(T)\right) \\
= & \int_{0}^{T}\left[\left\langle f^{\mathcal{O}}+\dot{\psi}_{h}, v\right\rangle-a\left(t ; v, \psi_{h}\right)\right] \mathrm{d} t
\end{aligned}
$$

since the approximation $\psi_{h}$ verifies the final condition $\psi_{h}(T)=u_{T}^{\mathcal{O}}$.

\section{References}

[1] S. Adjerid, J.E. Flaherty, and I. Babuška. A posteriori error estimation for the finite element method-of-lines solution of parabolic problems. Math. Models Methods Appl. Sci., 9(2):261-286, 1999.

[2] M. Ainsworth and J. T. Oden. A posteriori error estimation in finite element analysis. John Wiley \& Sons, Chichester, 2000.

[3] I. Babuška and S. Ohnimus. A posteriori error estimation for the semidiscrete finite element method of parabolic differential equations. Comput. Methods Appl. Mech. Engrg., 190(35-36):4691-4712, 2001. 
[4] I. Babuška and T. Strouboulis. The finite element method and its reliability. Numerical Mathematics and Scientific Computation. The Clarendon Press Oxford University Press, New York, 2001.

[5] P. Díez and G. Calderón. Goal-oriented error estimation for transient parabolic problems. Comput. Mech., electronic, 2007.

[6] K. Eriksson and C. Johnson. Adaptive finite element methods for parabolic problems. I. A linear model problem. SIAM J. Numer. Anal., 28(1):43-77, 1991.

[7] A. Huerta and P. Díez. Error estimation including pollution assessment for nonlinear finite element analysis. Comput. Methods Appl. Mech. Eng., 181(1-3):21-41, 2000.

[8] P. Ladevèze and D. Leguillon. Error estimate procedure in the finite element method and applications. SIAM J. Numer. Anal., 20(3):485-509, 1983.

[9] P. Ladevèze and J.P. Pelle. Mastering calculations in linear and nonlinear mechanics. Mechanical Engineering Series. Springer-Verlag, New York, 2005. Translated from the 2001 French original by Theofanis Strouboulis.

[10] L. Machiels. A posteriori finite element bounds for output functionals of discontinuous Galerkin discretizations of parabolic problems. Comput. Methods Appl. Mech. Engrg., 190(26-27):3401-3411, 2001.

[11] P. Morin, R.H. Nochetto, and K.G. Siebert. Local problems on stars: a posteriori error estimators, convergence, and performance. Math. Comp., 72(243):1067-1097, 2003.

[12] J. T. Oden and S. Prudhomme. Goal-oriented error estimation and adaptivity for the finite element method. Comput. Math. Appl., 41(5-6):735-756, 2001.

[13] M. Paraschivoiu, J. Peraire, and A. T. Patera. A posteriori finite element bounds for linearfunctional outputs of elliptic partial differential equations. Comput. Methods Appl. Mech. Engrg., 150(1-4):289-312, 1997. Symposium on Advances in Computational Mechanics, Vol. 2 (Austin, TX, 1997).

[14] N. Parés, J. Bonet, A. Huerta, and J. Peraire. The computation of bounds for linearfunctional outputs of weak solutions to the two-dimensional elasticity equations. Comput. Methods Appl. Mech. Engrg., 195(4-6):406-429, 2006.

[15] N. Parés, P. Díez, and A. Huerta. Bounds of functional outputs for parabolic problems. part i: Exact bounds of the discontinuous galerkin time discretization.

[16] N. Parés, P. Díez, and A. Huerta. Subdomain-based flux-free a posteriori error estimators. Comput. Methods Appl. Mech. Engrg., 195(4-6):297-323, 2006.

[17] A. M. Sauer-Budge, J. Bonet, A. Huerta, and J. Peraire. Computing bounds for linear functionals of exact weak solutions to Poisson's equation. SIAM J. Numer. Anal., 42(4):1610-1630 (electronic), 2004.

[18] A. M. Sauer-Budge and J. Peraire. Computing bounds for linear functionals of exact weak solutions to the advection-diffusion-reaction equation. SIAM J. Sci. Comput., 26(2):636652 (electronic), 2004. 
[19] T. Strouboulis, I. Babuška, and D. K. Datta. Guaranteed a posteriori error estimation for fully discrete solutions of parabolic problems. Internat. J. Numer. Methods Engrg., 56(9):1243-1259, 2003.

[20] V. Thomée. Galerkin finite element methods for parabolic problems, volume 25 of Springer Series in Computational Mathematics. Springer-Verlag, Berlin, second edition, 2006.

[21] T. Werder, K. Gerdes, D. Schötzau, and C. Schwab. hp-discontinuous Galerkin time stepping for parabolic problems. Comput. Methods Appl. Mech. Engrg., 190(49-50):66856708, 2001.

[22] Z. C. Xuan, N. Parés, and J. Peraire. Computing upper and lower bounds for the $J$-integral in two-dimensional linear elasticity. Comput. Methods Appl. Mech. Engrg., 195(4-6):430443, 2006. 